\title{
EL CONTROL DE LOS PRECIOS EXCESIVOS EN EL DERECHO DE LA LIBRE COMPETENCIA: ANÁLISIS Y PROPUESTA*
}

\author{
Eduardo Saavedra P. \\ Universidad Alberto Hurtado \\ Javier Tapia C. \\ Abogado
}

\begin{abstract}
Resumen: El control de los precios excesivos por autoridades de competencia ha sido objeto de mucha controversia a nivel global. En este artículo se argumenta a favor de tal control de una forma restrictiva, de modo que no conlleve un peligro para la innovación o las inversiones
\end{abstract}

Eduardo SaAvedra. PhD en economía por Cornell University. Profesor asociado del Departamento de Economía de la Universidad Alberto Hurtado. Ministro del Tribunal de Defensa de la Libre Competencia de Chile (TDLC). Email: saavedra@, uahurtado.cl.

JAVIER TAPIA. PhD en derecho por University College London y M.Sc. en regulación por la London School of Economics and Political Science. Ministro del TDLC. Email: jtapia02@gmail.com.

* Ésta es una versión bastante extendida y en español de Saavedra y Tapia (2017). Los autores señalan: "Agradecemos los comentarios de Claudio Agostini, Patrick Rey y Massimo Motta a versiones preliminares de este trabajo. Asimismo, algunos amigos en Latinoamérica nos ayudaron a revisar el estado actual del tema en sus respectivos países: Pamela Sittenfeld, Carlos Mena, Pablo Trevisán y Pablo Márquez. Dos árbitros anónimos y Ronald Fischer, co-editor adjunto de la publicación, también contribuyeron a enriquecer el trabajo, por lo que les estamos agradecidos. Finalmente, agradecemos igualmente los comentarios y opiniones de los panelistas y participantes del seminario "Precios excesivos en libre competencia", organizado por la Facultad de Economía y Negocios de la Universidad de Chile, en noviembre de 2018. Los errores (sobre todo los excesivos) son exclusivamente nuestros. Las opiniones aquí expresadas son estrictamente personales y no pretenden reflejar la visión de las instituciones en que trabajamos ni la de las demás personas que laboran en ellas". 
en I+D y sea compatible con diversos objetivos de la política de libre competencia. Luego de analizar la situación actual en Latinoamérica, se propone un test de aplicación más sencilla que los tradicionalmente utilizados en el derecho comparado, lo que permitiría superar los problemas prácticos tradicionalmente mencionados en la literatura y la jurisprudencia. El test consta de tres etapas. En primer lugar, se debe determinar si la firma acusada es "superdominante" en el mercado relevante, en cuyo caso se pasa a la etapa siguiente. En ésta se determina si el precio cobrado excede cierto umbral crítico de precios, el que corresponde al mayor valor entre un precio basado en costos y otro que impondría una empresa dominante con una participación de mercado igual al parámetro definido para la superdominancia. De superarse este umbral, en una tercera etapa la firma superdominante tendría la oportunidad de justificar su práctica de precios excesivos en, por ejemplo, la existencia de inversiones hundidas, el resultado de innovación o su definición como una empresa multiproducto o una plataforma.

PALABRAS ClAVE: precios excesivos, política de competencia, libre competencia, abuso de posición dominante.

RECIBIDO: agosto 2018: ACEPTADO: marzo 2019.

Clasificación JEL: K21; L13; L40.

\section{CONTROL OF EXCESSIVE PRICING IN COMPETITION LAW: ANALYSIS AND PROPOSAL}

ABSTRACT: The control of excessive pricing by competition authorities has been a matter of great controversy around the world. This article argues for a restricted form of such control so that it does not jeopardize innovation or investment in $R \& D$ and is compatible with various competition policy objectives. After analysing the current situation in Latin America, it proposes a test that is simpler to apply than those traditionally used in comparative law and would deal with the practical problems traditionally brought up in the literature and jurisprudence. The test is in three stages. The first step is to determine whether the accused firm is "super-dominant" in the market concerned. If it is, the next step is proceeded to: determining whether the price charged exceeds a certain critical price threshold, this being the greater of a cost-based price and the price that would be charged by a dominant firm with a market share equal to the parameter set for super-dominance. If this threshold is exceeded, the super-dominant firm will be given the opportunity to justify its excessive pricing as being due, for example, to the existence of sunk investments, to innovation or to its being a multi-product firm or a platform.

KEYwords: excessive pricing, competition policy, free competition, abuse of dominant position.

RECEIVED: August 2018: ACCEPTED: March 2019.

JEL Classification: K21; L13; L40. 


\section{INTRODUCCIÓN}

$\mathrm{L}^{2}$ a forma más intuitiva a través de la cual una firma dominante - esto es, aquella que posee un alto poder de mercado- puede obtener beneficios supra-normales es ejecutando una conducta que perjudique directamente el interés de los consumidores, sin producir ningún efecto en la estructura de competencia. A este tipo de prácticas se les denomina "explotativas" (Lyons 2007; Bishop y Walker 2010)․ Las más comunes consisten en discriminar precios de una forma relevante para la libre competencia o en cobrar por un producto un precio significativamente superior al que se estima competitivo. Este trabajo trata varios aspectos relacionados con este último tipo de conducta, denominada comúnmente de "precios excesivos". En particular, muestra el desarrollo doctrinario y jurisprudencial en la materia, con énfasis en la evolución que esta última ha tenido en el derecho chileno, para luego proponer una regla objetiva de análisis que permita determinar cuándo un precio debe ser considerado excesivo; esto es, una regla que no se basa en si un precio es "alto", sino en la diferencia entre el precio y dos benchmarks, o puntos de referencia alternativos: el precio que cubriría exactamente los costos medios de largo plazo de la firma y el precio de equilibrio en un mercado en que tal empresa es dominante y enfrenta baja competencia.

La necesidad del control de precios excesivos se justifica tanto si se adopta una perspectiva restrictiva como una perspectiva amplia de los objetivos subyacentes al derecho de la competencia. Por una parte, si se considera que el objetivo es la sola eficiencia económica, no lidiar con la práctica que lo afecta más directamente implica derechamente aceptar por anticipado una renuncia a su protección íntegra. ${ }^{2}$ Como es

${ }^{1}$ Las conductas explotativas se oponen a las "exclusorias", de mayor significancia para la libre competencia. En este segundo tipo de conductas, la intervención a la competencia se da por la vía de afectar rivales e, indirectamente, a los consumidores. Un buen tratamiento reciente de este tipo de conductas se encuentra en Fumagalli, Motta y Calcagno (2018).

2 Varias son las nociones de eficiencia económica (Motta 2004). Como es estándar en política de competencia, nuestro interés está en la eficiencia en la asignación de recursos y, por tanto, en evitar conductas que conlleven un nivel de producción menor al competitivo e impidan a oferentes y demandantes obtener todos los beneficios de intercambiar en el mercado. Con todo, también nos referimos puntualmente en el texto a la eficiencia dinámica; esto es, la capacidad del mercado para promover inversiones en el largo plazo y reducir progresivamente los costos de producción (por ejemplo, a través de inversiones en investigación y desarrollo). 
bien sabido, la noción de que los precios cobrados por sobre los costos - en particular por un monopolio- produce una pérdida de eficiencia en la asignación de recursos es parte del "A-B-C" de la economía. Por otra parte, adoptando una óptica más amplia, la tendencia actual es considerar al derecho de la competencia como una herramienta más —dentro del amplio espectro de políticas públicas - que permite lidiar con las desigualdades y promover la inclusión en todos los sectores de la economía, pues resulta evidente que el poder de mercado tiene un efecto directo en la desigualdad (por ejemplo, Baker y Salop 2015). Por esta sola razón, el control, o enforcement, de las prácticas explotativas debiera ser una prioridad.

Dado lo anterior, desde la perspectiva de la política pública, dicho control es una herramienta importante que debiera estar al servicio de las autoridades de competencia y ser empleada toda vez que se dé cumplimiento a ciertas condiciones, muy restrictivas. Esto es especialmente aplicable en el contexto latinoamericano y en otros países en vías de desarrollo. En muchos mercados la entrada es difícil debido a razones de facto, económicas o legales, y las firmas que eran otrora monopolios (legales o naturales) mantienen aún un alto poder de mercado. Si a esto se suma que la regulación es muchas veces débil o inexistente, la firma dominante tiene altos incentivos a comportarse de manera oportunista. De aquí que, para brindar una completa protección del interés de los consumidores, no resulte suficiente restringir la persecución de conductas anticompetitivas a las meras prácticas que afectan estructuras de mercado.

Con todo, como hemos adelantado, las condiciones para condenar este tipo de prácticas deben ser muy restrictivas, pues existen buenas razones para sostener que un uso indiscriminado o amplio del control podría producir precisamente el efecto contrario al buscado. Por la vía de privar a una firma que ha obtenido su posición en el mercado producto de sus inversiones en mejorar calidad o precios, sea innovando o mejorando su eficiencia productiva, de los beneficios legítimos que derivan de su actuación en el mercado, se desincentivaría a las demás firmas a desafiar dicha posición. Asimismo, un control excesivo de precios por parte de las autoridades de competencia iría en contra de principios de libertad básicos, en virtud de los cuales incluso una firma con alto poder de mercado puede cobrar por sus productos precios tan elevados como le sea posible dadas sus restricciones de demanda. 
Estos y otros argumentos han hecho que el uso del derecho de la competencia para controlar los precios excesivos sea controversial y menos aceptado que otras formas de abuso (particularmente, menos que las prácticas exclusorias). De hecho, por ejemplo, el derecho norteamericano no sanciona este tipo de prácticas. La mayoría de los países latinoamericanos, lamentablemente, tampoco las suele considerar en sus legislaciones o en su práctica. ${ }^{3}$ En este trabajo controvertimos varias de las premisas contenidas, implícita o explícitamente, en los argumentos que justifican esta práctica. Si el poder de mercado no siempre es producto de innovaciones, sino que también puede provenir del uso de un privilegio mal otorgado o prácticas ilícitas pasadas, y si el mercado no siempre tiene libre entrada, entonces resulta deseable y conveniente que la autoridad de libre competencia tenga las herramientas para discernir cuándo se está o no frente a precios excesivos abusivos.

Sin perjuicio de la discusión en torno a los argumentos que justifican un mayor control, gran parte de las legislaciones que sí lo aplican (de manera restringida, atendidas las aprensiones antedichas) enfocan el análisis no en la justicia - en abstracto - del precio cobrado, sino en el establecimiento de una medida que permita evaluar en concreto si un determinado precio es o no excesivo. De hecho, buena parte de la controversia en torno a la política de precios excesivos descansa en realidad en este aspecto práctico. Quizás por esto en algunas jurisdicciones la medida es definida legalmente. ${ }^{4}$ En la mayoría de los países, sin embargo, ella es determinada jurisprudencialmente $\mathrm{y}$, como veremos, en la práctica es común utilizar varios puntos de referencia y, por tanto, diferentes umbrales, sobre la base de los cuales la autoridad luego decide si el precio cobrado es o no excesivo.

Con todo, la vaguedad de algunos de los parámetros más utilizados, las dificultades en su aplicación o la posibilidad de que ellos den lugar a resultados disímiles han llevado a que el control de los precios excesivos sea complejo y, por ello, no alcance un lugar alto entre las prioridades de las autoridades.

Con el fin de superar estas dificultades, en este trabajo proponemos una regla única, objetiva y de fácil aplicación práctica, que per-

\footnotetext{
${ }^{3}$ Ver sección 2.2 .

${ }^{4}$ Según reportan Elhauge y Geradin (2011, 413-415), así ocurriría, por ejemplo, en Sudáfrica, Turquía y Corea del Sur.
} 
mite determinar cuándo un precio debiera ser considerado excesivo y que impone, al mismo tiempo, restricciones a las acusaciones temerarias a fin de evitar la sobre-litigación, minimizando de este modo el riesgo de afectar la eficiencia dinámica de la industria y desincentivar la innovación y las inversiones en innovación y desarrollo (I+D). En otras palabras, se trata de un test explícitamente "pro-defendido", destinado a actuar únicamente cuando un precio sea extremadamente alto.

Al igual que otros propuestos en la literatura (Evans y Padilla 2005; Motta y De Streel 2007), nuestro test consta de tres etapas. En la primera se determina si la firma es "superdominante"5. Luego, se establece si el precio efectivamente cobrado por ella está bajo o sobre un cierto umbral, el que - como adelantamos - corresponde al valor más alto entre dos precios alternativos: por un lado, el precio óptimo que resulta de un planificador central que buscar maximizar el bienestar total; y, por el otro, el precio de mercado en un equilibrio estático que cobraría una empresa dominante que posee una participación de mercado límite predefinida. Si el precio efectivamente cobrado está bajo dicho umbral, no debería ser revisado. Si, en cambio, está por sobre el umbral, es considerado prima facie excesivo. En la tercera etapa, la firma puede intentar justificar económicamente por qué el precio que ella cobra excede dicho nivel. A diferencia de otros modelos, en el nuestro sólo en la tercera etapa se consideran las posibles justificaciones basadas en innovación o inversión en $\mathrm{I}+\mathrm{D}$, entre otras.

El resto del trabajo se estructura de la siguiente manera. Con el objetivo de dar un marco teórico a la discusión, en la sección 2 mostramos brevemente cómo han sido analizados los precios excesivos en el derecho de la libre competencia, tanto a nivel doctrinario como en la práctica comparada. En la sección 3 se analiza detalladamente el caso chileno. Luego, en la sección 4, exponemos el test, distinguiendo cada una de sus tres etapas. Los detalles técnicos son mostrados en recuadros. Finalmente, la sección 5 concluye.

\footnotetext{
${ }^{5}$ En el sentido definido adelante. Ver nota 67 y el texto al que acompaña.
} 


\section{EL ANÁLISIS DE LOS PRECIOS EXCESIVOS: REVISIÓN DE LA SITUACIÓN MUNDIAL ACTUAL}

Tal como mostramos en esta sección, el control de los precios excesivos es de difícil aceptación en el mundo académico y entre autoridades de competencia. La razón principal dice relación con el hecho de que las rentas excesivas, incluso monopólicas, serían sólo —o principalmente - producto de inversiones previas que han llevado a dichas empresas a una situación de ventaja sobre sus rivales. Esta ventaja constituiría el premio al esfuerzo invertido y sería precisamente lo que las movilizó a realizar dichas inversiones en primer término. Luego, sería contrario a propiciar la innovación y el crecimiento castigar lo que es un resultado de inversiones exitosas. Adicionalmente, las propias rentas en un mercado abierto inducirían la entrada de nuevos rivales, los que, en definitiva, al competir con menores costos, reducirán las rentas, reestableciéndose un nuevo equilibrio con menores precios, mayor oferta y sin rentas extranormales para las empresas. ${ }^{6}$

Por el contrario, quienes están a favor de controlar los precios excesivos, aun reconociendo que ello debe hacerse bajo condiciones restrictivas, no comparten varias de las premisas contenidas, implícita o explícitamente, en el argumento descrito en el párrafo previo. En especial, debe reconocerse que el poder de mercado no siempre es producto de innovaciones, sino que puede provenir de otras fuentes, como por ejemplo el uso de un privilegio mal otorgado o de una práctica ilícita pasada, y que el mercado puede carecer de libre entrada. En estos casos, resulta socialmente deseable que la autoridad de libre competencia tenga las herramientas para discernir cuándo se está o no frente a precios excesivos abusivos.

\subsection{Algunas consideraciones doctrinarias}

Controlar los precios excesivos usando la política de competencia no es una idea muy aceptada entre muchos académicos. Esta práctica es vista como intervencionista; regulatoria, en una versión menos extrema;

${ }^{6}$ Nótese que la base de este argumento se encuentra en la hipótesis schumpeteriana de que, en el corto plazo, el monopolio genera rentas sobrenormales que incentivan la innovación, las que se disipan en el largo plazo producto de los nuevos entrantes (Schumpeter [1911] 1982). 
o, al menos, extremadamente difícil de implementar, ya sea por la dificultad de estimar el "precio correcto" o por los inadecuados remedios disponibles para resolver el problema (por ejemplo, Posner 1976; Motta 2004; Evans y Padilla 2005). Esto explica que la recomendación habitual sea que, "excepto en circunstancias excepcionales, las autoridades de competencia debieran usar sus facultades de persecución y sanción (enforcement) o de incidencia política (advocacy) para eliminar los obstáculos a la competencia que llevan a los precios supra-competitivos más que hacer uso del derecho de la competencia para establecer la ilicitud de los precios altos" (Jenny 2018, 5).

En primer término, ciertamente este control implica establecer cuál es el precio "correcto" sobre el cual realizar la medición. Esto, por cierto, depende de las concepciones económicas imperantes. Por ejemplo, la tradición escolástica y la política medieval europea consideraban el comercio como una relación entre personas y no como un mero intercambio de bienes, por lo que la noción de precio "justo" (fair o just), esencialmente basada en costos de producción, primaba en los análisis (Schumpeter [1954] 1996; Monsalve 2014; Hawk 2018). La idea sería reemplazada en el tiempo por el individualismo post-renacentista y luego por el mercantilismo, hasta llegar a la idea moderna de que el precio de mercado está basado en la oferta y la demanda y, en último término, en las preferencias del consumidor. ${ }^{7}$ Sólo con el desarrollo moderno de la organización industrial - mucho más tarde — serían agregados como requerimientos el que el precio deba estar "significativamente" y "persistentemente" sobre el nivel competitivo para ser considerado injusto. ${ }^{8}$

Hoy, es un hecho indiscutido en la disciplina económica que los precios cobrados por sobre los costos —en particular por un monopolioproducen una pérdida de eficiencia en la asignación de recursos (o "costo social", como es denominado en muchos textos de economía básica). Lo

${ }^{7}$ Modernamente, tanto la teoría marxista (de Marx y Engels) como los economistas clásicos (desde Smith) basarían sus teorías del valor en costos. Sería el neoclasicismo el que vincularía la noción de precio "justo" con el precio competitivo (especialmente a partir del marginalismo; por ejemplo, en Marshall [1890] 1997).

${ }^{8}$ En Europa estos conceptos han sido recogidos expresamente en la jurisprudencia (O’Donoghue y Padilla 2013, 755). En Chile, una discusión acerca del "precio natural" de los bienes o servicios se dio a propósito de la eventual aplicación del artículo 285 del Código Penal a materias de libre competencia (Salazar 2016). 
relevante, más allá de este desarrollo, es que, en la mayoría de los mercados, las firmas tienen la posibilidad y el incentivo para fijar sus precios más allá del costo de producción, pues poseen algún grado de poder de mercado a raíz de, entre otros, la diferenciación de productos, economías de escala o barreras de entrada. De aquí que en la práctica el análisis se deba centrar no en la justicia — en abstracto- del precio cobrado, sino en el establecimiento de una medida que permita evaluar en concreto si un determinado precio es o no excesivo. Como veremos, para estos efectos se ha propuesto una serie de parámetros (benchmarks) que permiten establecer cuándo un precio debe ser considerado excesivo.

\subsection{El control de los precios excesivos en el derecho comparado}

Distintas jurisdicciones tratan de manera disímil esta política. ${ }^{9}$ Como hemos visto, algunas la aceptan abiertamente como un ilícito antitrust en sí mismo, mientras que otras le niegan tal carácter. Entre las últimas se encuentra Estados Unidos, donde, de acuerdo con lo sostenido en una serie de precedentes jurisprudenciales, se ha considerado que el control de precios excesivos no es parte del antitrust, a menos que - eventualmente - sea parte de otra figura anticompetitiva. ${ }^{10}$ En breve, las razones fundamentales son que los precios excesivos incentivarían la entrada de otros actores, con lo cual en definitiva no se restringiría la cantidad total transada en el mercado, y que, siendo así, serían en realidad una suerte de premio a la firma que ha alcanzado un alto grado de poder de mercado gracias a sus ventas competitivas. ${ }^{11}$ Con todo, no es

${ }^{9}$ Para un resumen de las posturas "oficiales" de distintos países en la materia, ver Katsoulacos y Jenny (2018) y OECD (2011). El contraste entre Estados Unidos y Europa es presentado en Gal (2004).

${ }^{10}$ Los precedentes más relevantes son Berkey Photo Inc. v. Eastman Kodak Company 457 F. Sup 404 (S.D.N.Y., 1978); Berkey Photo Inc. v. Eastman Kodak Company 603 F.2d 263 (2nd Cir, 1979); In Re Air Passenger Computer Reservation Systems 727 F. Supp. 564 (C.D. Cal., 1989); Verizon Communications Inc. v. Law Offices Of Curtis V. Trinko, LLP 540 U.S. 398 (2004); In Re: Live Concert Antitrust Litigation 863 F. Supp. 2d 966 (C.D. Cal. 2012).

${ }^{11} \mathrm{Tal}$ y como fue formulado en Trinko: "The opportunity to charge monopoly prices — at least for a short period — is what attracts 'business acumen' in the first place; it induces risk taking that produces innovation and economic growth. To safeguard the incentive to innovate, the possession of monopoly power will not be found unlawful unless it is accompanied by an element of anticompetitive conduct" (Verizon Communications Inc. v. Law Offices of Curtis V. Trinko, LLP 540 U.S. 398 [2004], at 407). 
claro que exista un impedimento estrictamente legal para no aceptar el control de esta práctica. ${ }^{12}$

El derecho europeo se ubica en el otro extremo, pues los precios excesivos están expresamente incluidos en el Tratado de Funcionamiento de la Unión Europea (TFEU, por sus siglas en inglés) como una potencial conducta anticompetitiva. ${ }^{13}$ Los precedentes más importantes en la materia son Sirena/EDA (1970) ${ }^{14}$, General Motors $(1975)^{15}$, United Brands $(1978)^{16}$, British Leyland $(1986)^{17} \mathrm{y}$ AKKA/LAA $(2017)^{18}$, fallados por las Cortes, y Port of Helsingborg $(2004)^{19}$, decidido por la Comisión. Además, existe una serie de precedentes relacionados a nivel local que confirman esta importancia. ${ }^{20}$

12 Como recomiendan Baker y Salop (2015, 22 y ss.), los precios excesivos debieran ser reconocidos como parte del derecho de la competencia en Estados Unidos, sea mediante el uso de la Sección 5 de la FTC Act o incluso interpretando la propia Sherman Act.

${ }^{13}$ El artículo 102 del TFEU indica que, entre otros, son contrarios a la competencia los actos de una firma dominante cuya finalidad es "a) imponer directa o indirectamente precios de compra, de venta $\mathrm{u}$ otras condiciones de transacción no equitativas". Para la jurisprudencia europea, la expresión inequitativa (unfair) incluye tanto los precios predatorios como los excesivos. Ezrachi y Gilo (2009), entendiéndolo así, califican la política europea como "intervencionista".

${ }^{14}$ Caso 40/70, Sirena S.R.L. v. Eda S.R.L., [1971] ECR 69.

15 Caso 26/75, General Motors Continental NV v Commission of the European Communities, [1975] ECR 1367.

${ }^{16}$ Caso 27/76 United Brands v Commission [1978] ECR 207; [1978] 1 CMLR 429.

${ }^{17}$ Caso 226/84, British Leyland Public Limited Company $v$ Commission of the European Communities, [1984] ECR 3263.

18 Caso C177/16, Biedrība 'Autortiesību un komunicēšanās konsultāciju a'gentūra - Latvijas Autoru apvienība'v Konkurences padome (sin cita oficial a esta fecha). Para un comentario acerca de la misma, véase Leslie (2018).

${ }^{19}$ Caso COMP/A.36.568/D3, Scandlines Sverige AB v Port of Helsingborg, Decision of 23 July 2004 y caso COMP/A.36.568/D3, Sundbusserne v Port of Helsingborg, Decision of 23 July 2004.

${ }^{20}$ Entre otros, además de los antecedentes recientes mencionados en la nota anterior, ver: Napp Pharmaceutical Holdings Ltd. and others v Director General of Fair Trading, 69 BMRL 69 (Competition Commission Appeals Tribunal); Spótdzielnia Mieszkaniowa "Podzamcze" v Prezes Urzędu Ochrony Konkurencji $i$ Konsumentów, CASELEX:PL:2005:20 (Sąd Apelacyjny $w$ Warszawie, Poland, 2005); Van Ommeren Agencies Rotterdam B.V., Pakhoed Agencies Rotterdam B.V., Vertom Scheepvaart, en Handelmaatschappij B.V., Ruys \& CO. B.V.v De Gemeente Rotterdam (Gemeentelijk Havenbedrijf Rotterdam), AF2005 (Rechtbank Rotterdam, Netherlands, 2002); British American Tobacco Nederland B.V. v. Chipknip C.V. and 
Si bien la mayoría de los fallos son relativamente antiguos, el fallo del 2017 y otros antecedentes recientes parecen dar cuenta de un renovado interés en el tema. ${ }^{21}$

General Motors fue el primer caso en que la Corte de Justicia Europea (CJE) estableció que un precio podía ser abusivo si excedía el "valor económico" del servicio ofrecido. Luego, en United Brands —el caso europeo más relevante-, la CJE determinó que el precio que la empresa cobraba no tenía "relación razonable con el valor económico del producto ofrecido", ligando este concepto a los costos de producción y el precio de los productos competidores. Sin embargo, para

Ceanet B.V., CASELEX:NL:2008:26 (Gerechtshof Amsterdam, Burgerlijke Kamer, Netherlands); FAC Flygbussarna Airport Coaches AB and 5 others $v$ Konkurrensverket och Luftfartsverket, CASELEX:SE:2005:10 (Marknadsdomstolen, Sweden); BHB Enterprises plc v Victor Chandler (International) Limited, [2005] EWHC 1074 (Ch) (Chancery Division); The British Horseracing Board Ltd., BHB Enterprises plc v Attheraces Ltd., Attheraces (UK) Ltd., [2007] EWCA Civ 38 (Court of Appeal of England and Wales); The British Horseracing Board Ltd., BHB Enterprises plc $v$ Attheraces Ltd., Attheraces (UK) Ltd.

${ }^{21}$ Recientemente, varias autoridades europeas han lidiado con casos de precios excesivos. La mayoría han sido en el sector farmacéutico (ver por ejemplo, el reciente workshop del Grupo de Competencia de la Organización para la Cooperación y el Desarrollo Económicos, OECD, realizado el 28 de noviembre de 2018, disponible en: http://www.oecd.org/daf/competition/excessive-pricing-in-pharmaceuticals.htm). Entre ellos: Italia en 2016, contra Aspen (Autorità Garante della Concorrenza e del Mercato, "Price increases for cancer drugs up to 1500\%: the ICA imposes a 5 million Euro fine on the multinational Aspen", comunicado de prensa, 14 de octubre, 2016); UK en 2016, contra Pfizer y Flynn (Competition and Markets Authority, "Unfair pricing in respect of the supply of phenytoin sodium capsules in the UK", decisión del 7 de diciembre 2016, publicada en junio 2017), revertida por el Competition Appeal Tribunal con fecha 25 de julio de 2018 (decisión disponible en: https://www.catribunal.org.uk/sites/default/files/2018-08/1275-76_Flynn_Judgment_CAT_12_250718.pdf); la Comisión Europea en 2017 (European Commission, "Antitrust: Commission opens formal investigation into Aspen Pharma's pricing practices for cancer medicines", comunicado de prensa, 15 de mayo de 2017). En otros contextos, las reglas de precios excesivos han sido invocadas en disputas de licenciamiento de patentes sobre "estándares esenciales" (SEPs) (por ejemplo, en UK: Unwired Planet International v Huawei Technologies, [2017] EWHC 711 (Pat), judgment of 5 April 2017). Ver también la base de datos Concurrences, eCompetitions Bulletin Special Issue, June 2018 (http://www.concurrences.com/en/ bulletin/special-issues/excessive-prices/). 
efectos del cálculo, la CJE reconoció que es posible utilizar diversos comparadores. $^{22}$

Varias decisiones posteriores han utilizado un enfoque similar, estableciéndose así el test legal de dos etapas que rige hasta la actualidad. Éste consiste en comparar, por una parte, el costo real con el precio efectivamente cobrado (es decir, establecer el margen de ganancia) y, por otra, determinar si el precio es injusto en sí mismo o en comparación con los productos de los competidores (esto es, si es un precio “justo"). ${ }^{23}$ De manera reciente, en $A K K A / L A A$, la CJE agregó que, para ser excesiva, la diferencia entre el precio competitivo y el real debe ser "significativa" ("de cierta importancia", en la traducción oficial al español) y debe, además, "persistir durante un determinado período y no ser temporal o episódica" (párrafo 56). ${ }^{24}$

En otros países, fuera de Europa, las leyes de competencia también permiten sancionar precios excesivos como un abuso de dominancia, aunque las autoridades respectivas rara vez han atacado esta práctica. ${ }^{25}$ Un ejemplo es Sudáfrica, donde es la propia ley la que define que precio excesivo es aquel que no tiene relación con el valor económico del bien o servicio. ${ }^{26}$ Allí, el principal precedente es Harmong $v$ Mittal Steel (2007), el cual, sin embargo, concluyó en un acuerdo con la autoridad luego de que fuera fallado a favor de esta última por

22 United Brands, párr. 250-2. Véase también AKKA/LAA, opinión del abogado general Wahl, 6 de abril de 2017, párr. 36. Una propuesta reciente de implementación de este test se encuentra en Davis y Mani (2018).

${ }^{23}$ Ambos tests son acumulativos, desde lo sostenido en Port of Helsingborg. Asimismo, la segunda parte hace referencia a dos cuestiones independientes, desde lo sostenido en Athens International Airport.

${ }^{24}$ Sobre el origen de estos conceptos, ver sección 2.1. También resulta relevante la opinión del abogado general de la CJE Nils Wahl en el caso AKKA/LAA, citada en nota 22.

${ }^{25}$ Para el análisis de algunos casos, véase Ezrachi y Gilo (2009).

${ }^{26}$ A diferencia de la ley chilena, que establece una única disposición amplia, comprensiva de todas las conductas que se estiman anticompetitivas (ver sección 3 y nota 44), la ley sudafricana establece disposiciones específicas para cada conducta. Así, la sección 8(a) de la ley prohíbe a una firma dominante cobrar precios excesivos en detrimento de los consumidores, definiendo tal precio como aquel que no tiene relación con el valor económico del bien o servicio y es mayor que dicho valor (véase la sección 1(1)(ix) "Definiciones e interpretación"). El valor económico no es definido en la ley. Nótese que este criterio es similar al usado en el derecho europeo. 
el Tribunal de Competencia, pero revertida esta decisión por la Corte de Apelaciones de Competencia y enviado el caso de vuelta al tribunal aduciendo que éste no había calculado directamente el valor económico del servicio. ${ }^{27}$ Con todo, quizás lo más destacable para nuestros propósitos aquí es que el tribunal sudafricano efectuó centralmente un análisis de las circunstancias del mercado y consideró muy relevante para condenar el hecho el que, dadas sus altísimas ventajas estructurales, la firma poseía "superdominancia". ${ }^{28}$ Asimismo, es importante considerar que la legislación sudafricana requiere específicamente probar "detrimento al consumidor", cuestión compleja en la práctica cuando el afectado es una firma de tamaño pequeño. De aquí que, actualmente, un proyecto de reforma a la ley de competencia deja sin efecto esta prueba. ${ }^{29}$

Los países latinoamericanos se ubican en distintos planos. La mayoría de ellos son más proclives a no considerar, sea por ley o en la práctica, el control de precios excesivos como parte de la política de competencia (Márquez 2012). Con todo, muchos casos han sido analizados como casos de discriminación de precios (De León 2009). Por el contrario, otros países controlan expresamente los precios en exceso de cierta medida.

${ }^{27}$ El test usado por la Corte es extraño. Indica: "What the legislature must be taken to have intended by 'economic value' is the notional price of the good or service under assumed conditions of long-run competitive equilibrium. This requires the assumption that, in the long-run, firms could enter the industry in the event of a higher than normal rate of return on capital, or could leave the industry to avoid a lower than normal rate of return. It does not imply perfect competition in the short-run, but rather competition that would be effective enough in the long-run to eliminate what economists refer to as 'pure profit' - that is a reward of any factor of production in excess of the long-run competitive norm which is relevant to that industry or branch of production". Para un análisis más completo de este caso, véanse Roberts (2008) y Das Nair (2008).

28 Sobre la importancia de considerar las características del mercado, ver sección 4.1. En este sentido, de acuerdo con Das Nair (2008, 289), la definición de precio excesivo del tribunal fue: "Cuando aparece que el precio no tiene otra explicación que no sea el solo ejercicio de poder de mercado (como se muestra por la estructura del mercado y cualquier conducta secundaria relevante de parte de la firma dominante), entonces el precio no es razonable en relación con su valor económico" (traducción libre de los autores).

${ }^{29}$ Las propuestas y una nota explicativa pueden consultarse en https://www. gov.za/sites/default/files/41294_gon1345.pdf/. 
Ejemplos del primer grupo hay varios. El caso más extremo es Perú, donde la actual ley de competencia (Decreto Legislativo 1.034) establece expresamente que sólo califican como conductas de abuso aquéllas con efecto exclusorio. ${ }^{30}$ Ésta es una verdadera declaración de legalidad per se en la materia, totalmente excepcional al menos en el contexto latinoamericano. ${ }^{31}$ En México, la Ley Federal de Competencia no es tan explícita como la ley peruana, pero tampoco contempla la figura expresamente en su articulado. ${ }^{32}$ Como consecuencia, no han existido condenas basadas en ella. ${ }^{33}$ La situación es similar en Centroamérica. Por ejemplo, ni Costa Rica ni Nicaragua sancionan conductas abusivas por precios excesivos. ${ }^{34}$ Finalmente, en Brasil, la autoridad de

${ }^{30} \mathrm{La}$ ley establece, en su artículo 10.2: "El abuso de la posición de dominio en el mercado podrá consistir en conductas de efecto exclusorio tales como...". En el 10.5 dice: "No constituye abuso de posición de dominio el simple ejercicio de dicha posición sin afectar a competidores reales o potenciales".

${ }^{31}$ Sin embargo, en el pasado, bajo la vigencia de leyes anteriores, sí se conocieron casos por precios excesivos. Bajo el Decreto Legislativo 701, derogado por la ley actual, la normativa no era tan clara. En esa época se evaluó un caso importante, CUT y Diez Canseco v las AFP y la Asociación. En primera instancia se hizo un análisis bastante detallado, incluso desde un punto de vista constitucional, sobre por qué bajo el ordenamiento peruano no se deberían sancionar los precios excesivos y la denuncia debía ser desestimada (Decisión de Indecopi 054-2003-INDECOPI/CLC, recaída en el Expediente $\mathrm{N}^{\circ}$ 001-2003-CLC, de 10 de diciembre de 2003). Sin embargo, en segunda instancia la decisión fue revocada y se admitió la denuncia (Decisión del Tribunal de Defensa de la Competencia y de la Propiedad Intelectual, sala de defensa de la competencia, Resolución No 429/2005/TDC-INDECOPI, Expediente $\mathrm{N}^{\circ} 1-2003 /$ CLC).

32 Entre los supuestos para que una conducta sea abusiva (denominadas "prácticas monopolísticas relativas"), el artículo 54 de la Ley Federal de Competencia mexicana establece que dicha conducta se "encuadre en alguno de los supuestos a que se refiere el artículo 56 de esta Ley", el cual, a su vez, sólo establece conductas de tipo exclusorio.

${ }^{33}$ Bajo el imperio de la ley anterior, la autoridad de competencia, aunque no descartaba la posibilidad, estimaba que la política no era adecuada para economías en desarrollo, atendidos los altos costos y riesgos asociados a la aplicación del derecho de la competencia. Véase OECD (2011, 271 y ss.).

${ }^{34}$ Con todo, en un estudio reciente de la autoridad hondureña, en el mercado de tarjetas de crédito, se estableció que "las tarjetas de crédito muestran tasas de interés elevadas" y que los intereses no responden a una lógica de oferta y demanda, sino que son "antojadizos" y respondían más bien a una lógica del modelo de "líder-seguidor". Ver Comisión para la Defensa y Promoción de la Competencia (CDPC), "Análisis del mercado de las tarjetas de pago (crédito y débito) en Honduras", junio 2016. 
competencia - CADE, siglas de Consejo Administrativo de Defensa Económica- ha sostenido tradicionalmente que los precios excesivos sólo son una infracción a la normativa de competencia si tienen propósitos exclusorios. ${ }^{35}$ Históricamente, esta autoridad ha revisado más de 60 casos relativos a precios abusivos, la mayoría en el mercado farmacéutico, pero los ha descartado todos ante la ausencia de dicho propósito (Martínez 2013, 174).

Argentina se encuentra en una situación "incierta" en la actualidad, pues posee la ley de competencia más reciente aprobada en Latinoamérica (Ley 27.442 / 2018). Aunque no es clara, la ley no parece descartar del todo la sanción a la conducta de abuso. ${ }^{36}$ En la práctica, bajo el imperio de la ley anterior, muchos casos fueron tratados como relativos a discriminación, pero su base estuvo en la práctica de precios excesivos (Coloma 2009). ${ }^{37}$ Sin embargo, en un caso reciente (también fallado con la ley anterior), la autoridad de competencia acogió una denuncia en contra de la sociedad de autores y compositores, a cargo de fijar los aranceles por derechos de autor, mediante la cual fue acusada de subir unilateralmente dichos aranceles al sector hotelero "en base a tarifas irreales". La resolución estableció expresamente que "los aranceles excesivos para todo tipo de hotel, discriminatorios entre hoteles, y no razonables relacionados con la ejecución secundaria de música han resultado en perjuicio del interés económico general, por cuanto han directamente incrementado los costos

35 En teoría, los precios excesivos podrían ser sancionados legalmente bajo la actual Ley 12.529/2011. La anterior ley de competencia incluso incluía como ejemplo de práctica anticompetitiva el cobrar "precios abusivos, o el incremento no razonable del precio de un producto o servicio". El ejemplo, sin embargo, fue quitado en la actual ley (Art. 36), atendida la posición histórica del CADE, restringiendo aún más las posibilidades de aplicación.

${ }^{36}$ En efecto, mientras en su artículo $1^{\circ}$ establece: "Están prohibidos (...) los actos o conductas, de cualquier forma manifestados, relacionados con la producción e intercambio de bienes o servicios, que tengan por objeto o efecto limitar, restringir, falsear o distorsionar la competencia o el acceso al mercado o que constituyan abuso de una posición dominante en un mercado, de modo que pueda resultar perjuicio para el interés económico general", el artículo $3^{\circ}$ letra d) ejemplifica, indicando que es contrario a la competencia el "impedir, dificultar u obstaculizar a terceras personas la entrada o permanencia en un mercado o excluirlas de éste".

${ }^{37}$ Ver también Resolución CNDC No 189/99, Comisión Nacional de Defensa de la Libre Competencia v YPF S.A. (condenando) y Marcelo Larribite v Profertil S.A., Expediente 064-013453/2001 (justificando las diferencias de precios). 
del sector hotelero". ${ }^{38}$ En nuestra opinión, la nueva ley no debiera modificar en gran medida esta línea jurisprudencial.

En el otro lado del espectro, un caso de aceptación legal expresa de la conducta se presenta en Colombia. ${ }^{39}$ Allí los precios excesivos son sancionables en virtud de lo establecido en el artículo $1^{\circ}$ de la Ley 155 de 1959, que prohíbe las conductas tendientes a "mantener o determinar precios inequitativos". Sobre esta base, la autoridad de competencia - la Superintendencia de Industria y Comercio (SIC) ha investigado a una serie de empresas dominantes en diversos mercados. ${ }^{40}$ Quizás los casos más relevantes sean Vijagual $(2011)^{41}$ y $\operatorname{CASYP}(2014)^{42}$. En el primero, la autoridad indicó que el hecho de que un precio sea elevado no es el único factor a considerar, sino que también deben tenerse en cuenta las barreras de entrada excepcionales e insuperables existentes en el mercado. El caso terminó sin sanción, pues la SIC concluyó que las diferencias de precio respondían a distintas calidades del producto y condiciones geográficas del mercado. En CASYP, en cambio, la firma dominante - una concesionaria de aeropuertos - fue sancionada por fijación de precios inequitativos, pues, de acuerdo a la autoridad, no se tuvo en cuenta la situación comercial o financiera de la concesión para establecer las tarifas de acceso cobradas para abastecer a los distribuidores mayoristas de combustible en el aeropuerto, y no se utilizaron métodos transparentes para establecerlas. En su resolución, la SIC indicó que están prohibidas las conductas encaminadas a la determinación de un precio que "no tiene en cuenta las reglas del mercado y el libre juego de la oferta y la demanda", para lo cual se debe examinar el contexto

${ }^{38}$ Federación Empresaria Hotelera Gastronómica de la República Argentina (FEHGRA) v Sociedad Argentina de Autores y Compositores de Música (SADAIC), decisión de la Comisión Nacional de Defensa de la Competencia de fecha 17 de mayo de 2017, Dictamen CDNC No 43 (aún sin cita oficial). La Resolución del Secretario de Comercio es de fecha 26 de junio de 2018.

${ }^{39}$ Además del caso chileno, tratado en la sección 3.

${ }^{40}$ Para un análisis de la situación en Colombia, véase Márquez y Mutis (2018) (con énfasis en la industria aeroportuaria). Estos últimos autores sostienen que, aunque algunos casos han sido descartados, en otros la autoridad "ha actuado como un pseudo regulador al imponer límites de precios a los participantes dominantes del mercado".

${ }^{41}$ Resolución No. 37.790 de 2011.

42 Resolución No. 76.724 de 2014. 
del mercado y del producto en particular y establecer si se trata de una "práctica, procedimiento o sistema tendiente a fijar un precio artificialmente elevado". ${ }^{43}$

\section{EL CONTROL DE LOS PRECIOS EXCESIVOS EN CHILE}

En Chile, al igual que en Europa, no existe duda de que la legislación de defensa de la competencia (el DL 211) acepta la sanción de los abusos explotativos en general, incluyendo tanto la discriminación arbitraria de precios como los precios excesivos. En efecto, no sólo el inciso primero artículo $3^{\circ}$ es reconocido por ser un precepto abierto e inusualmente amplio - lo que se manifiesta tanto en la forma de consagrar el objetivo protegido, como en la manera de consagrar las conductas que se consideran contrarias a la competencia (Tapia 2014)—, sino también la letra "b" del inciso segundo establece expresamente la ilicitud de tal conducta dentro de los posibles abusos de posición dominante. ${ }^{44}$ Siendo así, en un caso concreto será necesario demostrar poder de mercado (dominancia) y cómo se configura la conducta específica, la cual puede, de todos modos, estar económicamente justificada.

Sin embargo, la aplicación práctica de la norma no ha estado exenta de dudas y controversias. Por una parte, cierta doctrina ha pretendido - de manera infundada a nuestro entender- excluir este control del derecho nacional (Menchaca 2011). Por otra, de modo similar a lo que acontece en otras jurisdicciones - y de manera correcta, a nuestro entender-, las autoridades de competencia nacionales han atacado los precios excesivos en muy pocas ocasiones. Sí se ha hecho, en cambio, un uso intensivo de la defensa contra la discriminación de precios, aunque la base de varios casos sea, en los hechos, un precio excesivo.

${ }^{43}$ Ibídem, hojas 40 y 76 . El caso es analizado a fondo en Márquez y Mutis (2018).

${ }^{44}$ El artículo $3^{\circ}$ letra b) del DL 211 indica que se considera anticompetitiva "la explotación abusiva por parte de un agente económico, o un conjunto de ellos, de una posición dominante en el mercado, fijando precios de compra o de venta, imponiendo a una venta la de otro producto, asignando zonas o cuotas de mercado o imponiendo a otros abusos semejantes". 


\subsection{La naciente jurisprudencia sobre conductas explotativas $(2007-2008)^{45}$}

Entre los años 2007 y 2008 fueron presentados y fallados los primeros casos relativos a conductas explotativas. Al igual que en otros países de la región, ellos se refieren principalmente a conductas de discriminación de precios, cuyo análisis económico subyacente es distinto del de precios excesivos. Sin embargo, establecen un primer estándar básico que luego sería utilizado para juzgar conductas explotativas en general: que el cobro esté fundado en costos. ${ }^{46}$

En efecto, en Atrex con SCL; GTD con EFE y FNE con Lan Airlines, el Tribunal de Defensa de la Libre Competencia (TDLC) determinó que existía discriminación de precios debido a que el monto que la firma dominante estaba cobrando no estaba basado en ninguna medida de costo relevante o simplemente no tenían fundamento alguno. ${ }^{47}$ Mirada en sí misma, la exigencia de que se plantee al menos un fundamento (de cualquier índole) para cobrar un determinado precio parece extremadamente básica y tiene base en la proscripción de discriminaciones arbitrarias. Lo interesante es

${ }^{45}$ La jurisprudencia analizada es la del TDLC. Con anterioridad, se dieron algunos casos referidos a precios excesivos ante la Comisión Preventiva y Resolutiva, cuyo análisis no hemos incorporado. Uno relevante, sin embargo, es Minera El Toqui con Empresa Portuaria Chacabuco (Dictamen de la Comisión Preventiva Central N¹255). En él, siguiendo la recomendación del fiscal nacional económico, no se consideró que el precio cobrado por la empresa portuaria era excesivo porque se encontraba bajo el umbral de precio que hubiera fijado una empresa eficiente. Otro caso relevante es Julio Dittborn y otros con Metro S.A. (Dictamen de la Comisión Preventiva Central $\mathrm{N}^{\circ} 1154$ ), en el cual a esta última se le acusó de abusar de su posición dominante al aplicar un alza de las tarifas sin justificación en costos. El primer considerando del dictamen desestima la acusación, coincidiendo con la opinión de la FNE, basado en que las tarifas de Metro "se encuentran muy por debajo de las que prevalecerían si, por ejemplo, se aplicaran a dicha empresa los criterios de tarificación que imperan respecto de otros servicios públicos, como los servicios sanitarios o la distribución eléctrica” (pp. 19 del dictamen).

${ }^{46} \mathrm{La}$ existencia de casos en los que se ha basado la condena en prácticas de discriminación puede quizás deberse a la influencia de uno de los primeros libros de libre competencia publicados en Chile durante la década de 1990, precisamente titulado "La discriminación arbitraria en el derecho económico" (Valdés 1992).

47 Atrex con SCL (sentencia 75/2008), c. 51; GTD con EFE (sentencia 76/2008), c. 49; FNE con Lan Airlines (sentencia 55/2007), c. 39. Un razonamiento similar, aunque en un caso basado en incumplimiento de resolución judicial, se encuentra en Demarco v Coinca, sentencia 37/2006. 
que esta jurisprudencia establece que ese fundamento no puede ser de cualquier tipo, sino que debe ser de carácter económico; específicamente, estar relacionado con algún costo relevante de la firma (cualquiera que éste sea).

El escalón subsecuente, no planteado como estándar en los fallos mencionados, es establecer cuál es el costo relevante que debe ser utilizado en el caso concreto. En materia de libre competencia, la relevancia de un tipo de costo por sobre otro depende de la conducta y suele ser discutido, discusión que en muchos casos es zanjada por la jurisprudencia. ${ }^{48}$

Sin embargo, CCS I (sentencia 56/2007), un caso posterior, parece alejarse de la base económica del razonamiento para encontrar apoyo en la "razonabilidad" del fundamento. En efecto, luego de concluir que la estructura tarifaria usada por la firma dominante "no tiene fundamento en costos" (c. 40), el TDLC sostuvo que de todos modos "existen razones económicas que permitirían justificar la estructura de tarifas por tramos de deuda" (c. 45), por lo que la conducta no fue estimada arbitraria o "carente de razón”. Extrañamente, no se mencionaron cuáles eran esas otras razones, limitándose el fallo a constatar que "la circunstancia de que sea económicamente razonable una estructura de tarifas creciente por tramo de deuda no permite descartar que esa estructura pueda ser abusiva, ya que no cualquier progresión es razonable" (c. 46). En principio, esto deja bastante espacio a la discrecionalidad judicial: será el tribunal el que, en un caso concreto, establezca qué estructura es razonable en base a criterios que no se mencionan. Sin embargo, el tribunal luego restringe aún más su propio estándar antes mencionado, al indicar que "no tiene antecedentes que permitan identificar cuál sería el escalonamiento de tarifas socialmente óptimo" (c. 46, énfasis agregado). Esto implica que la única estructura tarifaria aceptable como no discriminatoria es aquella que establece un escalonamiento socialmente óptimo.

${ }^{48}$ Por ejemplo, en materia de precios predatorios, la discusión típica recae en la medida de costos bajo la cual un precio podrá ser considerado predatorio. En Chile, es claro que tal medida es el costo medio evitable (ver GPS con Entel, sentencia $78 / 2008$, c. 3), en contraposición a otras jurisdicciones que utilizan el costo medio variable (por ejemplo, en Estados Unidos, donde se lo conoce como el "test Areeda-Turner"). 
Con todo, más allá de las particularidades de los casos, las decisiones parecen haber estado guiadas por su naturaleza relativamente "clara", lo que permitió su resolución de forma más o menos directa. En efecto, en dos de los tres casos decididos en el año 2008, la firma dominante vulneró de forma abierta los términos de los contratos que establecían su campo de acción. ${ }^{49}$ En el tercero, en tanto, las diferencias de precio eran enormes y recaían sobre dos productos (agua y alcantarillado) que estaban literalmente a uno y otro lado de la calle, con regulación aplicable en un lado, pero no en otro. ${ }^{50}$

\subsection{Emelat (2010) y la doctrina contraria a los precios excesivos}

El primer caso en el que específicamente se desarrolló el tema del control de precios excesivos fue Emelat (sentencia 93/2010). La Fiscalía Nacional Económica (FNE) requirió a una empresa concesionaria de distribución eléctrica por abuso de posición dominante en la prestación del "servicio de escolta para transporte de carga sobredimensionada" en sus zonas de concesión. Éste es un servicio no regulado que comprende tareas de programación e intervención en líneas de transmisión y distribución eléctrica (incluyendo la eventual interrupción del suministro), para permitir el paso de los vehículos que transportan carga cuya altura sea superior a la del tendido eléctrico. Sólo la empresa concesionaria puede realizar este servicio. Entre las conductas imputadas se encontraba el cobro por parte de la concesionaria de un precio estimado excesivo por dos servicios incluidos en la "escolta". ${ }^{51}$

El TDLC adoptó una interpretación reduccionista del DL 211 e indicó que cobrar precios excesivos no constituye una conducta abusiva en sí misma (c. 30), pues "no es correcto sostener que este Tribunal mediante el expediente de determinar, en un caso concreto, cuáles precios serían excesivos y cuáles no- se erija en un regulador de precios

49 FNE con Edelmag (sentencia 73/2008) y Atrex con SCL (sentencia $75 / 2008)$.

50 Constructora Independencia con Aguas Nuevo Sur Maule (sentencia $85 / 2009$ ). Nótese que en algunas jurisdicciones (como Sudáfrica, por ejemplo) este caso debiera haber sido presentado como precio excesivo, dadas las restricciones de la ley en materia de discriminación arbitraria de precios.

${ }^{51}$ En particular, el cobro de los ítems "indemnización a clientes por no suministro" y los "gastos generales, imprevistos, administración" (sic). 
a consumidores finales, pues dicha regulación sólo puede efectuarse por expresa disposición legal en mercados que así lo requieran..." (c. 31). No obstante, siguiendo la línea norteamericana, se dejó abierto un espacio a la sanción de precios excesivos, cuando éstos fueran consecuencia de otro acto abusivo: "Si la causa que permite a la empresa acusada cobrar estos precios, superiores a los que prevalecerían en un mercado competitivo, consiste en la existencia de hechos, actos o convenciones contrarios a la libre competencia que sean de su responsabilidad, dicha empresa puede y debe ser sancionada por tales conductas" (c. 32). De hecho, la propia sentencia menciona ejemplos: "La imposición artificial de barreras a la entrada, las prácticas exclusorias - incluyendo la fijación de precios excesivos a un insumo esencial para excluir competidores aguas abajo - y otras estrategias de precio tales como la discriminación arbitraria o la fijación de precios de reventa, o estrategias de ventas atadas, entre otras, que no tengan una justificación económica razonable" (c. 32). Nótese, sin embargo, que la vinculación con las conductas exclusorias es altamente indeterminada. De hecho, la sentencia no menciona cómo sería la vinculación de un precio excesivo con cada uno de los casos que pretenden ilustrarla.

En el caso concreto, el TDLC consideró que los cobros estaban justificados, pues o bien algunos de ellos estaban justificados económicamente (c. 41) o no se demostró su falta de justificación (c. 44). Como se observa, de esta sola formulación no queda claro en quién recae la carga de la prueba.

Sobre la base de los razonamientos anteriores, Emelat fue fallado en contra del demandante, quien recurrió ante la Corte Suprema. En un fallo conciso, la Corte no acogió la tesis reduccionista. ${ }^{52}$ Indicó con claridad que "una de las modalidades que puede adoptar el abuso de una posición monopólica es el cobro de precios excesivos que carecen de justificación económica" (c. 3).

Sin embargo, inmediatamente a continuación la sentencia se desvía por un instante de las consideraciones estrictamente económicas para determinar lo excesivo de un cobro y aplica un razonamiento de orden contractual: "Si dicho cobro injusto ha sido obtenido por una empresa que detenta tal posición por la presión que ello implica para el otro

${ }^{52}$ Sentencia rol No $1022-2010$, de fecha 18 de agosto de 2010. 
contratante —al carecer de la opción de elegir a otro prestador — debe ser sancionado en sede antimonopolio". Es decir, lo injusto radica en las presiones ejercidas por el contratante más poderoso al privar de una opción al otro más débil. ${ }^{53} \mathrm{El}$ razonamiento económico es retomado luego, al establecerse la dificultad probatoria de la conducta. Según indica la Corte, "el primer escollo es precisar costos de compleja cuantificación (...) para luego estimar la 'utilidad razonable' en el mismo mercado relevante en que incide" (c. 4). Es decir, la Corte hace alusión a la necesidad de determinar el margen precio/costos y la utilidad para establecer si un precio es o no excesivo, lo que es consistente con los tests utilizados en el derecho comparado.

En definitiva, la Corte confirmó el fallo de primera instancia, pues la FNE no probó la falta de justificación de los cobros efectuados por la empresa eléctrica de una forma económica, en particular, aludiendo a los costos. En palabras de la Corte, la FNE “debió proponer algún parámetro de comparación para determinar la concurrencia de ese exceso, vale decir, acreditar la cuantía de los costos que le acarreaba a la empresa eléctrica el proporcionar el servicio de escolta que le fuera requerido..." (c. 5). El argumento, entonces, fue de hecho, no de derecho: es posible revisar la práctica, pero no fue posible controlar que ella haya sido ejecutada efectivamente.

No obstante, las consecuencias prácticas de Emelat fueron negativas. Durante los siguientes cuatro años no fue presentado ni un solo caso basado en el cobro de precios excesivos. ${ }^{54} \mathrm{La}$ tendencia sólo sería revertida en el año 2014, con el caso conocido como Campomar. $^{55}$

${ }^{53}$ Este es un punto interesante que, sin embargo, excede los límites de este trabajo. Aun cuando no es correcto centrar el razonamiento en la perspectiva contractual, la "elección del consumidor" puede ser un objetivo de la libre competencia distinto del más comúnmente mencionado "bienestar del consumidor", que, a su vez, se vincula con la eficiencia económica. Véase, por ejemplo, Averitt y Lande (2007).

${ }^{54}$ Es innegable lo difícil de establecer causalidad en esta materia. Si la FNE o los privados no presentaron casos porque ellos fueron desincentivados por la nueva tendencia es un tema que permanecerá irresuelto. Pero Emelat bien puede haber sido, al menos, causa concurrente o remota. Nótese que en PTLA (sentencia 100/2010) la empresa dominante fue acusada de imponer tarifas "excesivas". Sin embargo, el TDLC determinó que los cobros no excedían lo autorizado por los contratos aplicables, aunque se referían a servicios que no estaban siendo efectivamente prestados, lo cual era en sí abusivo.

55 Ver sección 3.3. 
Como se observa, Emelat es un fallo con un contenido más ideológico que los anteriores, los cuales carecían de declaraciones generales acerca de las conductas explotativas y se limitaban a juzgar los hechos en cada caso concreto. En un sentido, el cambio es entonces positivo, pues se establece una regla de actuación más clara para los agentes económicos. Sin embargo, no son claras las razones por las cuales la incipiente jurisprudencia, que sí aceptaba las sanciones en materia de conductas explotativas, varió de manera tan drástica (el fallo mismo no trata las razones del cambio), más aún cuando la composición del TDLC hasta entonces no había sufrido variaciones ${ }^{56}$ y la mayoría relativa en cada caso estuvo compuesta esencialmente por los mismos integrantes, como se observa en la tabla 1.

Sólo uno de los jueces de esa época ha expresado públicamente su posición en la materia. De manera prácticamente coincidente con Emelat, el entonces presidente del TDLC publicó un trabajo en el que defendió con firmeza y ahondó en los argumentos subyacentes a la tesis del fallo (Menchaca 2011). ${ }^{57}$ Con firmeza, ha expresado una similar opinión en fallos posteriores (Campomar, analizado en sección 3.3) y en discursos públicos. ${ }^{58}$ $\mathrm{Su}$ argumento puede resumirse en la afirmación de que "el tribunal no es, ni debe ser, un ente regulador de precios", el cual es precisamente

${ }^{56}$ Los ministros del TDLC duran seis años en su cargo y pueden ser reelegidos para un segundo período de igual duración

${ }^{57}$ En un capítulo, por ejemplo, argumenta que "no debiera sancionarse el mero hecho de que una empresa, incluso monopólica, fije precios altos, sin perjuicio de que ello pueda indicar su poder de mercado y la posibilidad de abusar del mismo y sin perjuicio de la posibilidad de recurrir a la regulación, incluso de precios, en caso de tratarse de un monopolio natural con gran poder de mercado, si es que tal regulación se justifica económicamente" (Menchaca 2011, 263). Asimismo, agrega dos argumentos. Primero que el beneficio de condenar tales prácticas sería muy bajo y los costos muy altos. Segundo, que existiría un riesgo de abrir la puerta a un tipo de regulación "que destruiría las bases esenciales del sistema de libre mercado" (Menchaca 2011, 264). Nótese que esta opinión sigue de cerca a la expresada por el juez Scalia, de la Corte Suprema de Estados Unidos, en el caso Trinko (ver nota 11).

${ }^{58}$ Por ejemplo, en la presentación de que da cuenta el sitio web: http://derecho.uc.cl/en/noticias/12313-icuando-los-precios-son-discriminatorios-o-excesivos (última visita 13.11.18). 
Tabla 1. COMPOSICIÓN DEL TDLC EN CASOS REFERIDOS A CONDUCTAS EXPLOTATIVAS (2007-2010)

\begin{tabular}{|c|c|c|c|c|c|c|c|c|}
\hline & Jara & Menchaca & Depolo & Butelmann & Peña & $\begin{array}{c}\text { Domper } \\
\text { (S) }\end{array}$ & $\begin{array}{c}\text { Palumbo } \\
\text { (S) }\end{array}$ & Prevención \\
\hline $\begin{array}{l}\text { Emelat } \\
\text { (sent } \\
93 / 2010 \text { ) }\end{array}$ & $x$ & $x$ & $x$ & & $x$ & $x$ & & \\
\hline $\begin{array}{l}\text { PTLA } \\
\text { (sent } \\
100 / 2010 \text { ) }\end{array}$ & & $x$ & & $x$ & $x$ & & & $\begin{array}{l}\text { ministra } \\
\text { Butel- } \\
\text { mann* }\end{array}$ \\
\hline $\begin{array}{l}\text { Aguas Nuevo } \\
\text { Sur } \\
\text { (sent } \\
85 / 2009 \text { ) }\end{array}$ & $x$ & $x$ & $x$ & $x$ & $x$ & & & \\
\hline $\begin{array}{l}\text { Edelmag } \\
\text { (sent } \\
73 / 2008 \text { ) }\end{array}$ & $x$ & & $x$ & $x$ & $x$ & & & \\
\hline $\begin{array}{l}\text { Lan } \\
\text { (sent } \\
55 / 2007 \text { ) }\end{array}$ & $x$ & & $x$ & $x$ & $x$ & & $x$ & \\
\hline $\begin{array}{l}\text { Atrex/SCL } \\
\text { (sent } \\
75 / 2008 \text { ) }\end{array}$ & $x$ & $x$ & $x$ & $x$ & $x$ & & & \\
\hline $\begin{array}{l}\text { CCS } \\
\text { (sent } \\
56 / 2007 \text { ) }\end{array}$ & & $x$ & $x$ & $x$ & $x$ & & & \\
\hline
\end{tabular}

* La prevención en este caso sólo se refirió a la multa aplicable.

Fuente: Elaboración propia.

el tipo de razonamiento dado en cierta jurisprudencia norteamericana y expresado en Emelat. ${ }^{59}$

Este tipo de razonamiento, sin embargo, nos parece infundado. Primero, entre un regulador de precios y una autoridad de competencia que sanciona por precios excesivos hay una amplia diferencia, pero ciertamente no en términos de capacidades. Además, el argumento aún resulta menos aplicable en presencia de un ente especializado como el

${ }^{59}$ Lamentablemente, el sólo hecho de sus afirmaciones permite únicamente dar una explicación meramente especulativa del cambio jurisprudencial, ligada a la posible influencia personal o valórica de algunos jueces. Esto se sustenta en alguna literatura comparada (por ejemplo, Cahill-O'Callaghan 2013). 
tribunal chileno, a diferencia del caso de Estados Unidos, donde quienes fallan los casos son cortes generalistas. Visto desde esta perspectiva institucional, se trata meramente de una cuestión de (auto)restricción en el uso de las competencias legales, mas no de un tema de expertise. Por otra parte, la hipótesis tampoco se sostiene desde una perspectiva meramente textualista. Si bien es cierto que pueden existir razones atendibles que hagan que atacar precios excesivos sea una opción poco atractiva para una agencia de competencia, lo cierto es que el argumento reduccionista es poco sostenible bajo el actual marco legal chileno. Como hemos visto, éste se refiere expresamente a la necesidad de evitar la "explotación" abusiva de una posición de dominio. De hecho, en países donde existe una redacción similar de la norma, como en las traducciones francesa y alemana del TFEU, ${ }^{60}$ la discusión se dio en algún momento respecto de la situación inversa: se preguntaba si la norma era aplicable sólo a abusos explotativos o si también podía ser extendida a abusos exclusorios, zanjándose finalmente (como es razonable) en favor de la interpretación amplia. ${ }^{61}$ En Estados Unidos, donde la sanción de precios excesivos no es aceptada, el texto legal es completamente diferente: se refiere a "monopolización", sin hacer mención a ninguna de las dos categorías típicas de abuso. En otras palabras, se sanciona la práctica tendiente a aumentar la participación de mercado afectando, con ello, ilegalmente a empresas rivales.

\subsection{Campomar (2014) y el enfoque favorable a los precios excesivos}

En Campomar (sentencia 140/14), un grupo de propietarios de terrenos de un condominio demandó ante el TDLC al desarrollador inmobiliario del condominio, argumentando que éste estaba abusando de su posición de dominio al cobrarles precios excesivos por el abastecimiento de agua potable, la cual era proveída a través de un estanque mane-

${ }^{60}$ El artículo 102 del Tratado de la Unión Europea se refiere a "...d'exploiter de façon abusive", en francés, y "mißbräuchliche Ausnutzung", en alemán. De modo similar, la norma española también habla de "explotación abusiva". El texto inglés, en cambio, sólo indica "any abuse... of a dominant position".

${ }^{61} \mathrm{La}$ situación fue resuelta por la Corte Europea de Justicia en favor de la interpretación amplia en Continental Can (caso 6/72, Europemballage Corp. and Continental Can Co. Inc. v. Commission [1973] CMLR 199). 
jado por el desarrollador. La demanda fue rechazada, pero la sentencia estableció una serie de aspectos relevantes.

Ante todo, Campomar estableció expresamente que los precios excesivos son un ilícito de acuerdo con el DL 211. La decisión del TDLC, sin embargo, no fue unánime, con dos ministros argumentando que la figura de precios excesivos no es un delito bajo la ley chilena. ${ }^{62}$

Enseguida, a efectos de implementar la decisión de mayoría, el tribunal aplicó el test de tres etapas establecido en United Brands ${ }^{63}$ (aunque sin mencionar de manera explícita la tercera), pero el requerimiento fue rechazado porque los propietarios de los terrenos tenían alternativas razonables de aprovisionamiento de agua potable y porque no se encontró evidencia de barreras de entrada al mercado relevante. En otras palabras, la dominancia no fue probada, por lo que no fue necesario establecer uno o más tests para determinar si en los hechos hubo o no precios excesivos.

La Corte Suprema finalmente confirmó la sentencia. ${ }^{64}$

\section{UNA REGLA OBJETIVA PARA ESTABLECER CUÁNDO UN PRECIO ES EXCESIVO}

En esta sección proponemos una forma específica de analizar los casos vinculados al control de precios excesivos. Nuestra premisa es que cualquier test que se utilice debe considerar condiciones para analizar la conducta de una manera restrictiva, pues, como hemos señalado, la postura contraria podría tener efectos negativos en la competencia, debido al riesgo de que estos casos puedan desincentivar la innovación y la eficiencia dinámica en la industria.

Siguiendo la doctrina establecida en Campomar, que es consistente a su vez con la jurisprudencia y doctrina europeas, proponemos un test dividido en tres etapas. En la primera se establece si la firma posee o no dominancia; esto es, un alto grado de poder de mercado. Consecuente

${ }^{62}$ Los dos autores de este trabajo estuvimos en la decisión de mayoría. Manteniendo su línea argumentativa (descrita en sección 3.2), en la minoría votó el ministro Menchaca, quien fue acompañado por la ministra Domper, quien también votó en Emelat (en ese entonces en calidad de ministra suplente).

${ }^{63}$ Ver sección 2.2 .

${ }^{64}$ Ver Vásquez (2015) para un análisis más detallado de esta sentencia. 
con nuestro enfoque restrictivo, en el test dicho poder debe ser particularmente alto y, por tal razón, lo hemos denominado "superdominancia". La segunda etapa determina un umbral concreto sobre el cual un precio es considerado prima facie excesivo ${ }^{65}$. En ambas fases la carga de la prueba recae sobre la parte demandante o acusadora (por ejemplo, la agencia de competencia). Finalmente, la tercera etapa tiene por objetivo dar la oportunidad a la empresa superdominante de presentar argumentos que justifiquen su práctica de precios.

\subsection{Primera etapa: analizando la dominancia para establecer "superdominancia"}

Como cualquier práctica unilateral, el análisis de precios excesivos debe comenzar por una definición del mercado relevante y su estructura, con el fin de determinar, luego, si existen barreras de entrada al mismo. El objetivo último de esto es establecer si una firma posee una posición de dominio en dicho mercado. En principio, esto no debiera ser diferente de cualquier otro caso de abuso de dominancia, sea exclusorio o explotativo. Lo relevante es determinar si el demandante posee alternativas factibles para los productos o servicios que ofrece la empresa dominante.

Sin embargo, el análisis de mercado posee particular relevancia en materia de precios excesivos. Una prohibición de estos precios sólo tendrá sentido económico si no existe la posibilidad de que las propias fuerzas del mercado remuevan o disminuyan de manera relevante la posición de dominio. En otras palabras, en un mercado competitivo no hay real posibilidad de controlar esta práctica, pues los precios altos efectivamente atraen nuevos entrantes y el problema se autocorrige.

Por lo anterior, ya en esta primera etapa del test es posible reducir el alcance del control en sede de competencia, siendo más estricto en el análisis de este tipo de casos que en aquéllos relativos a otros abusos de dominancia. Esto es posible de realizar, en esta etapa, por dos vías complementarias. Primero, evaluando estrictamente las barreras de entrada al mercado. Es decir, aspectos que podrían ser considerados barreras en un caso de abuso exclusorio pueden llegar a no serlo en un caso de precio excesivo.

${ }^{65}$ La idea de establecer si un precio es prima facie excesivo es utilizada también por Hou (2011), aunque de una forma muy distinta a la que aquí proponemos. 
La segunda vía de hacer más estricto el examen consiste en elevar el umbral de dominancia requerido. En Chile, siguiendo el criterio europeo, la jurisprudencia normalmente ha presumido que una firma que posee en torno al 50 por ciento de participación de mercado posee dominancia. ${ }^{66}$ En casos de precios excesivos, en cambio, dicho umbral debiera ser establecido en un porcentaje más alto, por ejemplo, en el 75 por ciento de participación. De aquí que pueda decirse, con algo de impropiedad en el lenguaje, que en este último tipo de casos debiera buscarse una suerte de "superdominancia" 67

Por lo anterior, para efectos de este trabajo y la aplicación de la segunda etapa del test, hemos definido la superdominancia precisamente utilizando un límite mínimo de 75 por ciento de participación de mercado.

Finalmente, debe notarse que, a diferencia de otros autores - como Motta y De Streel (2007) u O'Donoghue y Padilla (2013) O $^{68}$, nuestro test no verifica en esta etapa si la superdominancia proviene de innovaciones o inversiones en $\mathrm{I}+\mathrm{D}$ o si éstas tienen algún rol en el mercado. El análisis se centra únicamente en la participación de mercado de las empresas y la existencia de altas barreras de entrada, de modo que los consumidores no tienen alternativas creíbles con las que puedan reemplazar los productos de la empresa superdominante. ${ }^{69}$

66 Ver, por ejemplo, sentencia 161/2018, TVI $v$ VTR, c. 30, y sentencia 151/2016, Silcosil v Masisa, c. 26. En Europa este criterio está establecido en AKZO (caso C 62/86, AKZO v Commission [1991] ECR I-3359, [1993] 5 CMLR 215).

${ }^{67}$ Respecto de este punto, nuestro enfoque es similar a las condiciones 1 y 2 propuestas por Motta y de Streel $(2007,22)$. La "impropiedad" lingüística proviene del hecho que, en materia de libre competencia, el término "superdominancia" es de difícil definición o derechamente no está aceptado en muchas jurisdicciones (incluida la chilena). Con todo, aquí lo utilizamos principalmente relacionado con participaciones de mercado y con el propósito exclusivo de resumir en una palabra la necesidad de ser más estrictos en la definición de dominancia cuando se trata de casos de precios excesivos.

${ }^{68}$ O’Donoghue y Padilla $(2013,776)$ indican que la investigación de las políticas de precios de una firma dominante sólo debe continuar si "las firmas compiten en un ambiente maduro, donde las inversiones y la innovación juegan un rol pequeño o ninguno".

69 Sólo una vez superadas las dos primeras etapas, destinadas a determinar prima facie si un precio es excesivo, el peso de la prueba debe cambiar hacia la empresa acusada, a fin de que sea ésta la que justifique los precios cobrados que exceden el umbral, justificación que precisamente podrá estar basada en innovación, inversiones pasadas o una política comercial más amplia que sólo la de los productos o servicios acusados, entre otras razones (ver sección 4.3). 


\subsection{Segunda etapa: determinando el umbral de precios excesivos}

Una vez establecido que una empresa en el mercado es superdominante, es necesario analizar su conducta de precios a la luz de la normativa de libre competencia. Como hemos visto, desde la perspectiva económica es posible contrastar si los precios son excesivamente altos considerando diversos puntos de referencia, o benchmarks. Entre los más comunes se encuentran el margen de precios sobre alguna medida de su costo promedio (generalmente el variable), o la comparación con precios históricamente cobrados por la empresa (o cobrados por ésta a diferentes tipos de consumidores). Todos estos puntos de referencia han sido utilizados indistintamente por la jurisprudencia.

Asimismo, cada uno de estos métodos está sujeto a críticas que hacen que no sea recomendable su uso por separado. Por ello, es común que se exhorte su utilización de manera combinada, en tanto el benchmark sea razonable y provea de información útil sobre el precio bajo análisis y su posición relativa vis-à-vis el precio competitivo. ${ }^{70}$

Sin embargo, hasta donde sabemos, no existe en la literatura una propuesta concreta que especifique cómo tal combinación de benchmarks debiera ser ejecutada en la práctica. Con el objetivo de llenar ese vacío, en esta sección proponemos una forma que permite establecer un único umbral, de modo que el precio que la empresa superdominante realmente cobra pueda ser considerado prima facie competitivo o excesivo, dependiendo de si está por debajo o sobre tal umbral.

Como hemos adelantado, el umbral que proponemos es el valor máximo entre dos alternativas de precios. Por un lado, el umbral o precio límite debe al menos permitir que una eventual empresa monopólica regulada cubra sus costos medios de largo plazo. ${ }^{71} \mathrm{~A}$ este precio lo denominaremos $p^{*}$. Por otro lado, dicho precio límite no debe, adicionalmente, ser inferior al que cobraría una empresa dominante (para nuestros efectos, una que tiene menos del 75 por ciento de participación de mercado) como resultado de la interdependencia estratégica en un

${ }^{70}$ Este fue el método sugerido en Chile en el caso Campomar, en el Reino Unido para el caso NAPP, y por AG Wahl en su opinión ya mencionada para el caso en Lituania. Ver sección 2. Entre la doctrina, véase O’Donoghue y Padilla (2013, 776).

${ }^{71}$ Antecedentes del uso de este tipo de parámetro se encuentran en los casos de la Comisión Preventiva Central citado en nota 45. 
mercado oligopólico, cuando no hay reparos en sede de libre competencia por este precio. A este precio lo denominamos $p^{d}$.

De modo más formal, el umbral de precios máximos permitidos es

$$
\text { umbral }=\max \left\{p^{*}, p^{d}\right\}
$$

Analicemos ambos precios por separado, considerando por simpleza un ambiente estático.

El primer precio que compone el umbral propuesto corresponde teóricamente al precio óptimo que resuelve un problema de optimización de un planificador utilitarista que maximiza el bienestar total. Para estos efectos, con el objetivo de arribar a una solución simple, suponemos que la empresa produce un único bien y que cobra precios lineales. Este precio, $p^{*}$, dados los supuestos mencionados, corresponde a la suma de los costos promedios variables, fijos y hundidos de la empresa superdominante, como muestra la ecuación 1:

$$
p^{*}=c_{\text {avg }}+F_{\text {avg }}
$$

donde $c_{\text {avg }}$ son los costos promedios variables y $F_{\text {avg }}$ son los costos promedios fijos y hundidos de esta empresa superdominante. ${ }^{72}$ En otras

72 Nótese que la simplificación proviene, en primer término, del hecho de que otras consideraciones adicionales relacionadas con complejidades específicas que pueden presentarse en ciertos mercados (por ejemplo, si se trata de una empresa multiproductora o una que enfrenta competencia en sólo algunos de sus mercados) son tratadas en la tercera etapa del test, pues su prueba corresponde a la empresa superdominante. De modo similar, el supuesto de que a la empresa se le fijaría un precio lineal, el que la literatura denomina "precio lineal óptimo" (Joskow 2010, sección 6.1), es también una simplificación. Este precio es, en rigor, una solución de segundo mejor, ya que $p^{*}$ es mayor al costo marginal y, por tanto, genera ineficiencia en la asignación de recursos. $\mathrm{Si}$, por el contrario, la empresa cobrara tarifas en dos partes y conociera las disposiciones a pagar de sus diferentes tipos de consumidores, dicha estructura de precios sería óptima. Nos parece que casos como éste, que son más bien la excepción y no la regla, deben ser parte de la tercera etapa del test, en la que la prueba corresponde a la firma superdominante. Por último, también la tercera etapa está destinada a situaciones como traer a valor presente los costos medios de largo plazo de la empresa. 
palabras, este precio produce que los beneficios de largo plazo de la empresa sean iguales a cero.

Lo relevante de la ecuación es su simpleza, pues la información requerida para su estimación es de fácil acceso. En efecto, en la práctica es posible obtener proxies adecuados de los costos de empresas relativamente grandes, pues ellas son normalmente sociedades anónimas cuya contabilidad es auditada. Los costos operacionales o de explotación incluyen tanto a los costos variables como a los fijos, aunque los costos hundidos son típicamente desconocidos y no pueden ser extraídos directamente de la contabilidad de la empresa. Para solucionar este problema, un proxy razonable de los costos hundidos podría ser el valor anual de las inversiones; esto es, el costo de capital de la industria multiplicado por el valor de las inversiones capitalizadas en los estados financieros de la empresa superdominante. Por lo tanto, se podría considerar que:

$$
c_{\text {avg }}+F_{\text {avg }} \approx \text { Costos de Explotación }+k o \cdot \text { Inversiones }
$$

donde $k o$ corresponde al costo de capital de la empresa y es la única variable que requiere ser estimada o tomada de un benchmark (por ejemplo, del mismo mercado en otro país comparable). ${ }^{73}$

El segundo precio que compone el umbral propuesto es el precio que una empresa dominante debería cobrar en ese mercado en un equilibrio estático o de corto plazo, cuando dicha empresa posee exactamente el 75 por ciento de participación de mercado. Este precio, $p^{d}$, depende de parámetros económicos relacionados con el modelo de competencia que sea elegido.

Es relevante analizar, entonces, qué tipos de modelos podrían generar un equilibrio tal que una empresa posea tres cuartos del mercado y sus rivales, el cuarto restante. Analicemos cuatro opciones posibles.

La primera es el modelo de competencia à la Cournot, con bienes homogéneos. En este caso, es condición necesaria para el funcionamiento del modelo el que la empresa dominante haya adquirido su ventaja por el hecho de tener costos marginales muy inferiores a los de sus

${ }^{73}$ Alternativamente, una medida de $p^{*}$ puede ser también el costo incremental de desarrollo de largo plazo, muy utilizado en industrias con fuerte desarrollo tecnológico y demanda creciente, como las de telecomunicaciones. 
rivales. De hecho, suponiendo sólo dos empresas (la dominante y una rival), este modelo predice que la empresa pequeña debiera tener costos marginales muy superiores a los de la dominante, lo que hace que de por sí sea cuestionable y poco intuitivo en mercados con interacción estratégica y donde todas las empresas tienen acceso a las diversas tecnologías de producción. ${ }^{74}$

Una crítica similar puede hacerse a los modelos tipo Cournot en los que los consumidores tengan preferencias a priori por una empresa. Ellos podrían ser muy específicos a ciertas industrias y, por tanto, se requeriría explicar por qué los consumidores presentan ciertos sesgos en sus preferencias.

Una segunda opción la constituye un grupo de modelos de tipo Bertrand, con bienes diferenciados. Sin embargo, en ellos nuevamente se requiere que la explicación principal del equilibrio asimétrico sea o bien una diferencia muy alta en los costos marginales de las firmas, o diferencias a priori en las preferencias de los consumidores por una determinada firma, o bien, en este caso, parámetros de sustitución también asimétricos entre consumir los bienes producidos por un vis-à-vis la otra. Con todo, utilizando estos modelos es posible generar participaciones de mercado con el 75 por ciento para la empresa dominante, pero se requieren supuestos ad hoc a cada mercado, lo que no aconseja su empleo para la construcción de un benchmark de uso general.

Una tercera opción es considerar que se está en presencia de entrada reciente en una industria otrora regulada o con dominio de una empresa incumbente, como podría ser, por ejemplo, la industria de telecomunicaciones (en la mayor parte de las jurisdicciones occidentales). Al suponer un modelo à la Hotelling de bienes diferenciados, diversos autores muestran que es posible explicar la existencia de una empresa con el 75 por ciento o más de participación de mercado en el ámbito de la telefonía fija hacia principios de $\operatorname{los} 2000 .{ }^{75}$ Con todo, nuevamente

${ }^{74}$ Por ejemplo, para el caso de costos marginales constantes, si la elasticidad de la demanda fuera igual a 1 , los costos de la empresa más ineficiente deberían ser tres veces mayores que los de la dominante para generar una participación de mercado de la empresa dominante del 75 por ciento. Este mayor costo debería ser de 40 por ciento cuando dicha elasticidad es igual a 2 y de 22 por ciento cuando ella es igual a 3 .

75 Ver, por ejemplo, Carter y Wright (2003), utilizando un parámetro ad hoc de preferencia hacia el incumbente, y Mancero y Saavedra (2006), suponiendo una entrada estratégica en un mercado de reciente liberalización. 
el problema de estos modelos es que parecen ser muy propios de la industria específica $-\mathrm{O}$, al menos, específico de industrias que fueron reguladas-, no siendo generalizables sus conclusiones a mercados oligopólicos que mantienen su estructura en el largo plazo y donde una de las firmas es dominante.

Y la cuarta opción nos parece aplicable de modo más general a muchos más mercados. Ésta es la opción conocida como "modelo de empresa dominante" o "modelo de liderazgo de precios" de una empresa dominante. Este modelo, de común uso en casos de libre competencia, tal como lo señalan Landes y Posner (1981) y Kaplow y Shapiro (2010), data de principios del siglo pasado y es considerado por diversos autores (Deneckere y Kovenock 1992; Rassenti y Wilson 2004) como adecuado para ser usado como benchmark de mercados oligopólicos con participaciones de mercado asimétricas. ${ }^{76}$

Si bien existen muchas variantes del modelo de empresa dominante, hemos optado por utilizar el que explícitamente trata la interacción entre una empresa dominante y muchos rivales de menor tamaño que se comportan como tomadores de precios, siguiendo a Ono (1982). Según se explica en más detalle en el recuadro 1 , hemos asumido que una firma es dominante (no superdominante) en el margen que previamente hemos establecido; es decir, posee exactamente el 75 por ciento de participación de mercado. Como hemos explicado, en este caso la empresa debiera tener libertad para cobrar cualquier precio, sin importar cuál es la fuente de su alto poder de mercado. Para mostrar esta idea de una forma sencilla, supongamos que las demás empresas competidoras en este mercado (denominadas conjuntamente el fringe, o borde competitivo) son menos eficientes, por ejemplo, porque utilizan una tecnología diferente, no lineal. En este caso, la firma dominante actúa como monopolio de la demanda residual y cobra un precio que en un equilibrio estático está dado por la ecuación 2:

$$
p^{d}=c^{\prime} \cdot\left[\frac{\eta+\frac{1}{4} \cdot \varepsilon}{\eta+\frac{1}{4} \cdot \varepsilon-\frac{3}{4}}\right]
$$

${ }^{76}$ Con todo, si resultara que existe un modelo de competencia diferente al de liderazgo de precios que es más adecuado para el análisis de un caso concreto, en nuestro marco analítico la empresa (super)dominante siempre podrá utilizarlo en su defensa durante la tercera etapa del test que estamos proponiendo. 
donde $c$ ' es el costo marginal de la dominante, es la elasticidad de la demanda y es la elasticidad de oferta del fringe. ${ }^{77}$

Una posible crítica al uso de este modelo dice relación con el hecho de que utiliza costos marginales, los cuales — podría argumentarse- son difíciles de estimar (ciertamente más difícil que tener una buena aproximación de los costos medios de la firma). Con todo, existen diversas opciones que vuelven factible tal estimación. Una primera posibilidad es utilizar los costos medios variables, los que en general son mayores que los costos marginales cuando hay costos fijos o hundidos importantes. Una segunda forma de estimación de los costos marginales consiste en utilizar los costos incrementales de desarrollo de corto plazo, los que a su vez se pueden estimar a partir de información de costos de la empresa o de otras similares a ella. Una tercera opción consiste en estimar la función de costos totales, también a partir de información de la empresa o de otras similares, para luego obtener los costos marginales a partir de su derivada evaluada en la producción observada. ${ }^{78}$ Una cuarta opción es utilizar la condición de primer orden del problema de maximización de una empresa $y$, si se dispone de su participación de mercado y de su elasticidad de demanda, se obtiene el costo marginal por despeje. ${ }^{79}$ Finalmente, se puede obtener información de costos marginales estimados para mercados similares en otros países. Estas opciones muestran que, aun siendo difícil, es posible obtener una estimación

${ }^{77}$ Nótese que en equilibrio debe cumplirse que $\eta>1-\frac{1}{4} \bullet(1+\varepsilon)$. Esto implica que el rango aceptable de elasticidad de la demanda podría caer en la parte inelástica de la misma. Es claro que mientras más grande sea la elasticidad de oferta de los rivales de la empresa dominante (del fringe competitivo), más baja debería ser la elasticidad de demanda que se observe en equilibrio en un mercado que opere de la manera descrita en el modelo de firma dominante. Por ello, en equilibrio, la demanda de mercado puede ser tanto elástica como inelástica.

${ }^{78}$ Davis y Garcés (2010, cap. 3) muestran que es posible utilizar diversas metodologías para estimar econométricamente los costos marginales de una empresa, incluyendo las mencionadas en el texto principal. Al respecto, ver además los trabajos allí citados.

${ }^{79}$ La condición de primer orden de toda empresa $i$ en un mercado oligopólico es $\frac{p_{i}-c_{i}^{\prime}}{p_{i}}=\frac{1}{\eta_{i}}$, donde $\mathrm{p}_{\mathrm{i}}, \mathrm{c}_{\mathrm{i}}{ }_{\mathrm{i}} \mathrm{y} \mathrm{h}_{\mathrm{i}}$ son respectivamente su precio, costo marginal y la elasticidad de demanda por su producto. Luego, $c_{i}^{\prime}=p_{i}\left[\frac{\eta_{i}-1}{\eta_{i}}\right]$. Si se desconoce $\eta_{i}$, pero se conoce la elasticidad de demanda de mercado (además de la participación de esta empresa y la elasticidad de oferta del fringe), la fórmula debe ser ajustada. Por ejemplo, en el modelo de empresa dominante será $c_{i}^{\prime}=p^{d}\left[1-\frac{1-s}{\eta+s \cdot \varepsilon}\right]$. 
Recuadro 1. PRECIO DE EQUILIBRIO EN EL MODELO DE EMPRESA DOMINANTE

Suponemos un mercado con una demanda $Q(P)$, por supuesto decreciente en $\mathrm{P}$, cuya oferta la satisfacen dos tipos de empresas: la empresa dominante, que por simpleza suponemos que tiene costos marginales constantes iguales a c', y un gran número empresas pequeñas que son tomadoras de precios y suponemos que tienen una función de costos marginales crecientes con su producción, lo que en términos de precio se puede expresar como una función creciente $X(P)$. A este gran número de competidores le llamamos el fringe competitivo. La empresa dominante sabe que mientras más alto fije el precio sobre sus costos, de más oferta dispondrán sus rivales, por lo que la demanda residual que enfrenta la empresa dominante es $D(P) \equiv Q(P)-X(P)$. Luego, el problema de la empresa dominante es encontrar el precio óptimo que maximiza sus beneficios:

$$
\operatorname{Max}_{P} \quad\left(P-c^{\prime}\right) \cdot[Q(P)-X(P)]
$$

la condición de primer orden $(C P O)$ es:

$$
\left[Q\left(p^{d}\right)-X\left(p^{d}\right)\right]+\left(p^{d}-c^{\prime}\right) \cdot\left[Q^{\prime}\left(p^{d}\right)-X^{\prime}\left(p^{d}\right)\right]=0
$$

Todas las funciones son evaluadas en el nivel de precio óptimo que resuelve este problema, al que llamamos $p^{d}$. Al restar a ambos lados el primer paréntesis cuadrado y luego dividiendo por $p^{d}$ y por el último paréntesis cuadrado tenemos:

$$
\frac{\left(p^{d}-c^{\prime}\right)}{p^{d}}=\frac{-\left[Q\left(p^{d}\right)-X\left(p^{d}\right)\right]}{\left[Q^{\prime}\left(p^{d}\right) \cdot p^{d}-X^{\prime}\left(p^{d}\right) \cdot p^{d}\right]}
$$

Sean $s \equiv \frac{X\left(p^{d}\right)}{Q\left(p^{d}\right)}$ la participación de mercado del fringe, $\eta \equiv-Q^{\prime}\left(p^{d}\right) \frac{p^{d}}{Q\left(p^{d}\right)}$ la elasticidad de la demanda de mercado, y $\varepsilon \equiv X^{\prime}\left(p^{d}\right) \frac{p^{d}}{X\left(p^{d}\right)}$ la elasticidad de oferta del fringe, la expresión anterior es en equilibrio igual a:

$$
\frac{\left(p^{d}-c^{\prime}\right)}{p^{d}}=\frac{1-s}{\eta+s \cdot \varepsilon}
$$

Esto es, el índice de Lerner es igual a la participación de mercado de la empresa dominante divida por la elasticidad de la demanda residual que enfrenta esta misma empresa.

Luego de algo de álgebra, el precio que cobraría esta empresa cuando tiene un (1-s) de participación de mercado es:

$$
p^{d}=c^{\prime} \cdot\left[\frac{\eta+s \cdot \varepsilon}{\eta+s \cdot \varepsilon-(1-s)}\right]
$$

Si (1-s) es el 75 por ciento, es decir el límite exacto en que se consideraría lícito cobrar este precio, éste en equilibrio es:

$p^{d}=c^{\prime} \cdot\left[\frac{\eta+\frac{1}{4} \cdot \varepsilon}{\eta+\frac{1}{4} \cdot \varepsilon-\frac{3}{4}}\right]$

Esto es, el precio cobrado en equilibrio por una empresa dominante depende de las elasticidades de demanda de mercado y de oferta de sus rivales. 
Figura 1. EXPLICACIÓN GRÁFICA DEL UMBRAL PROPUESTO

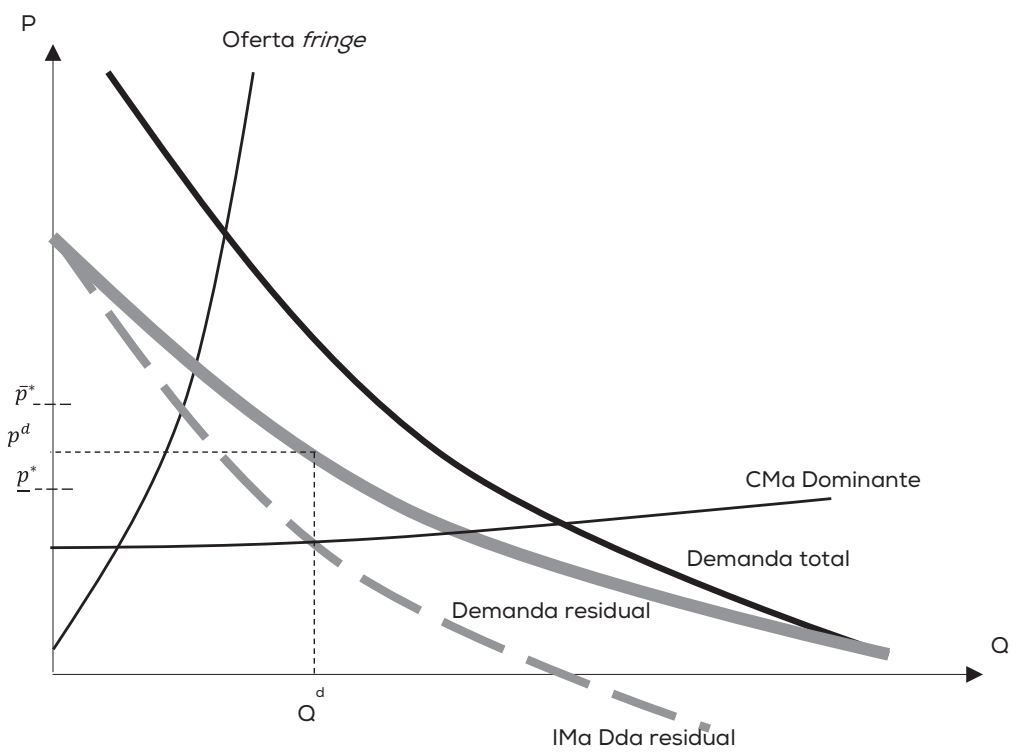

- Empresa dominante (participación hasta 75\%) fija el precio al igualar su costo marginal con el ingreso marginal de la demanda residual: cobra $p^{d}$, vende $Q^{d}$ y sus rivales completan la demanda a dicho precio.

- Empresa superdominante (cualquier participación mayor que $75 \%$ ) está sujeta al nivel de sus costos medios de largo plazo, $p^{*}$ :

- Si $p^{*}>p^{d}$, entonces el umbral es $p^{*}$ [caso $p^{*}=\bar{p}^{*}$ ]

- Si $p^{*}<p^{d}$, entonces el umbral es $p^{d}$ [caso $p^{*}=p^{*}$ ]

Fuente: Elaboración propia a partir de recuadro 1.

de los costos marginales de la empresa acusada para ser usada en la etapa 2 de este test, quedando en poder de la empresa acusada utilizar su propia información en la etapa 3 si considera que los costos marginales usados en su contra son irreales.

Volvamos ahora a centrarnos en el umbral de precios propuesto. Recuérdese que un precio será considerado excesivo sólo cuando esté por sobre dicho umbral, el cual es, a su vez, el valor mayor entre los dos precios $-p^{*} \mathrm{y} p p^{d}$ — antes analizados.

Dado que ambos precios dependen de diferentes parámetros, lo más probable es que ellos difieran en su valor. Atendido el límite de partici- 
Recuadro 2. EJEMPLO QUE EXPLICA EL UMBRAL PARA TEST DE PRECIOS EXCESIVOS

Supóngase que en la realidad la empresa superdominante tiene el 85 por ciento del mercado relevante, siendo el 15 por ciento restante para sus competidores, que son tomadores de precios. Usando los parámetros de Kahai et al. (1996) para el mercado de telefonía de larga distancia en Estados Unidos para los años 80 y 90, la elasticidad de demanda es de 0,49y la de la oferta de sus competidores, de 4,4.

Como consideramos una participación de mercado de 75 por ciento como límite para definir superdominancia, si suponemos un costo marginal para esta empresa igual a uno, obtenemos un valor $p^{d}=1,89$.

Por otro lado, si suponemos por simpleza que la empresa superdominante tiene un costo promedio variable también igual a uno, y si además el 70 por ciento de los costos de esta empresa son variables, entonces $p^{*}=1,43$.

Es claro que $p^{d}$ es mayor que $p^{*}$. Si el precio realmente cobrado por la empresa superdominante está entre ambos números (digamos, 1,68), tal precio no puede ser considerado como excesivo, pues si la empresa en realidad tuviese una participación de mercado bajo el 75 por ciento, el precio de 1,89 hubiese sido considerado como legalmente válido. En suma, el precio de 1,68 no puede ser considerado como ilegal, cualquiera sea la participación de mercado real de la empresa acusada.

pación de mercado que hemos propuesto ( 75 por ciento), bajo el cual la firma dominante puede cobrar libremente $p^{d}$ por su producto o servicio (aun cuando este precio esté muy por encima de sus costos marginales), la comparación deberá normalmente ser hecha entre el precio realmente cobrado y $p^{*}$. Sólo excepcionalmente, cuando este último valor $p^{*}$ sea inferior a $p^{d}$, este último cobrará relevancia. De otro modo, un precio efectivo incluso inferior a $p^{d}$ podría ser considerado legal hasta el 75 por ciento de participación de mercado, pero ilegal sobre dicha participación, si resulta que el precio efectivo supera a $p^{*}$. Tal situación sería no sólo injusta, sino que también redundaría en una asimetría regulatoria.

Una explicación gráfica del umbral propuesto, que resulta de suponer que la estructura del mercado es de una empresa dominante, se muestra en la figura 1. Un ejemplo que permite clarificar nuestra idea es desarrollado en el recuadro 2 .

Considerando, entonces, que la decisión de usar $p^{d}$ en lugar de $p^{*}$ como umbral debería ser correcta si y sólo si $p^{d}>p^{*}$; y dado que la elasticidad de oferta del fringe es generalmente desconocida, es posible 
utilizar algo de álgebra para imponer esta desigualdad en las ecuaciones 1 y 2 y llegar así a la siguiente restricción:

$$
\varepsilon \leq \frac{(1-s)}{s} \cdot \frac{1}{\varphi}-\frac{1}{s} \cdot \eta
$$

donde $\varphi \equiv \frac{\text { Favg }}{\text { Cavg }+ \text { Favg }}$ corresponde a la proporción de costos fijos y hundidos a costos totales de la empresa superdominante. ${ }^{80}$

Como puede observarse en la tabla 2, el valor resultante para la elasticidad de oferta del fringe que iguala ambos lados de la ecuación anterior es altamente dependiente del porcentaje de costos fijos y hundidos de la empresa dominante. Este resultado es relevante, ya que si la alternativa a utilizar información pública, o valores que son fácilmente deducibles de información públicamente disponible, es utilizar información privada de más difícil acceso, los resultados de la tabla 2 facilitan el análisis. En efecto, esta tabla muestra que si la mayor proporción de costos de la firma es variable (15 por ciento de costos fijos y hundidos), la comparación correcta será entre el precio realmente cobrado y $p^{d}$, ya que es improbable que la elasticidad de oferta del fringe sea mayor a cuatro (véase la primera columna desde la izquierda). En la medida en que los costos fijos y hundidos son más importantes, utilizar $p^{d}$ en lugar de $p^{*}$ requiere demostrar que la demanda total es esencialmente inelástica. Finalmente, en aquellos casos en los que los costos fijos y hundidos son más relevantes (por ejemplo, el 60 por ciento de los costos totales), como podría ser en industrias de infraestructuras y redes de servicios, es mucho más probable que $p^{*}$ sea el umbral correcto de utilizar (véase la columna en el extremo derecho de la tabla). En consecuencia, sólo aquellos casos mostrados en las celdas de color gris requerirían de un análisis más profundo. En particular, en dichos casos se requerirá estimar la elasticidad de oferta del fringe, para así determinar si el umbral correspondiente es $p^{d}$ o $p^{*}$.

Finalmente, analicemos el caso en que la comparación correcta debiera ser hecha entre el precio efectivamente cobrado y el umbral dado

${ }^{80}$ Esta ecuación supone que la razón entre los costos marginal y promedio variable es igual a uno, lo que simplifica el resultado, pero no debiera variar la argumentación. De no ser iguales, $\varphi \equiv \frac{\left(c_{\text {avg }}-c^{\prime}\right)+\text { Favg }}{\text { Cavg }+ \text { Favg }}$, por lo que el valor límite de la elasticidad de oferta del fringe no varía mayormente en tanto $c_{\text {avg }} \approx c^{\prime}$. 
Tabla 2. VALOR MÁXIMO DE LA ELASTICIDAD DE OFERTA DEL FRINGE (PARA USAR $p^{d}$ EN LUGAR DE $p^{*}$ )

\begin{tabular}{l|rrrr}
\hline Elasticidad de demanda & \multicolumn{4}{c}{ \% Costos fijos y hundidos sobre costos totales } \\
\cline { 2 - 5 } & $15 \%$ & $30 \%$ & $45 \%$ & $60 \%$ \\
\hline 0,5 & 18,00 & 8,00 & 4,67 & 3,00 \\
1 & 16,00 & 6,00 & 2,67 & 1,00 \\
1,5 & 14,00 & 4,00 & 0,67 & negativa \\
2 & 12,00 & 2,00 & negativa & negativa \\
2,5 & 10,00 & 0,00 & negativa & negativa \\
3 & 8,00 & negativa & negativa & negativa \\
\hline
\end{tabular}

Fuente: Elaboración propia.

por $p^{d}$, y que los costos variables promedio y marginales son iguales. Bajo estas condiciones, la razón entre el umbral y el costo variable promedio depende de las elasticidades de demanda y de oferta del fringe. Esta razón se muestra en la tabla 3 y la figura 2, para algunos valores de aquélla. Para efectos comparativos, también mostramos los valores que cobraría un monopolista que maximiza beneficios y el que resulta en caso de competencia à la Cournot con costos asimétricos y una firma dominante que tiene el 75 por ciento de participación de mercado.

Como se observa, para niveles bajos de elasticidad de demanda de mercado (bajo 1,5), el precio sobre el costo marginal cobrado por un monopolista es sustancialmente mayor que el sobreprecio que cobraría una firma dominante que, en el margen, pasaría a ser superdominante (es decir, con una participación de mercado de 75 por ciento). Esta diferencia se incrementa en la medida en que la elasticidad de oferta del fringe es más elástica. Por el contrario, cuando las demandas son más elásticas (sobre 2), hay muy poca diferencia entre el sobreprecio que cobraría un monopolio maximizador de beneficios y el sobreprecio aceptable en un mercado oligopólico, cualquiera sea la elasticidad de oferta del fringe. De este resultado se deriva que los casos relevantes en materia de precios excesivos debieran darse en mercados con elasticidad de demanda media o baja, mas no en aquellos casos en que el mercado presenta elasticidades de demanda muy altas. La intuición detrás de este resultado es que una alta elasticidad de demanda refleja la existencia de muy buenos sustitutos, lo que deja pocos espacios para que la empresa pueda cobrar precios 
Tabla 3. MÁXIMA RAZÓN $p / c^{\prime}$ (SOBREPRECIO) ACEPTABLE

\begin{tabular}{lc|rrrrr}
\hline Elasticidad de & $\begin{array}{c}\text { Sobreprecio del } \\
\text { demanda }\end{array}$ & \multicolumn{5}{|c}{ Elasticidad de oferta del fringe } \\
\cline { 3 - 7 } & monopolio & 0 * & 1 & 2 & 3 & 4 \\
\hline 1 & $\infty$ & 4,00 & 2,50 & 2,00 & 1,75 & 1,60 \\
1,5 & 3,00 & 2,00 & 1,75 & 1,60 & 1,50 & 1,43 \\
2 & 2,00 & 1,60 & 1,50 & 1,43 & 1,38 & 1,33 \\
2,5 & 1,67 & 1,43 & 1,38 & 1,33 & 1,30 & 1,27 \\
3 & 1,50 & 1,33 & 1,30 & 1,27 & 1,25 & 1,23 \\
\hline
\end{tabular}

*: Modelo de Cournot que explica participación de mercado de 75 por ciento por asimetrias en costos marginales.

Fuente: Elaboración propia.

Figura 2. MÁXIMO SOBREPRECIO ACEPTABLE PARA UNA EMPRESA DOMINANTE

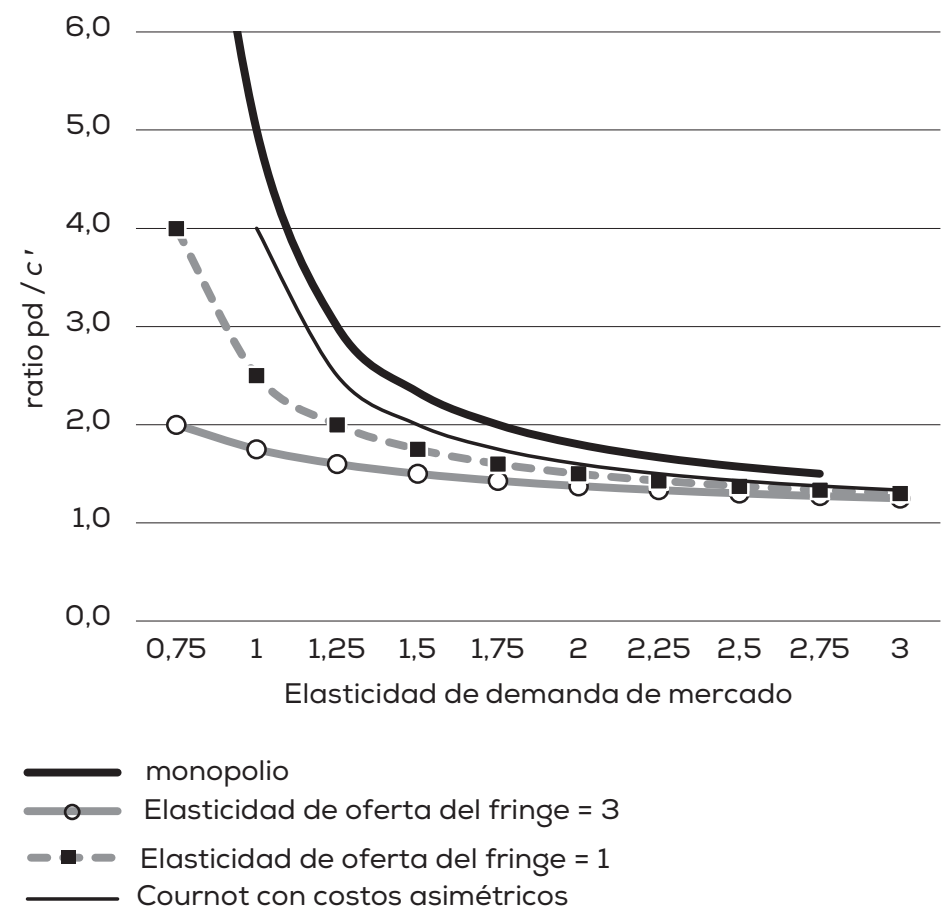

Fuente: Estimaciones propias a partir de la tabla 3. 
excesivos, aun cuando ella sea superdominante considerando su participación de mercado, ya que un alza desmedida en sus precios llevará un desvío de su demanda hacia esos sustitutos cercanos.

\subsection{Tercera etapa: consideración de posibles defensas}

En términos generales, los precios excesivos no deberían ser castigados en sede de libre competencia cuando ellos reflejen razonablemente innovaciones o inversiones riesgosas realizadas en el pasado. Por lo tanto, una vez que ha sido establecido prima facie que un precio es excesivo, la firma superdominante debe tener la oportunidad de justificar por qué está cobrando precios que superan el umbral, sea que éste corresponda a $p^{d}$ o $p^{*}$, según se ha visto. En la tercera etapa de nuestro test, entonces, el peso de la prueba le corresponde al acusado.

No es posible establecer, por cierto, un catálogo cerrado de posibles justificaciones. A continuación explicamos a modo ejemplar tres posibles razones y analizamos su plausibilidad.

Una primera justificación está relacionada con la valorización de inversiones fijas y hundidas realizadas por la empresa en el pasado. En esta línea, la firma podría argumentar, por ejemplo, que sus inversiones en activos fijos no están adecuadamente recogidas por los métodos de contabilidad IFRS, cualquiera sea la razón subyacente a ello; que realizó inversiones en $\mathrm{I}+\mathrm{D}$ que aún no han madurado, por lo que no dispone de patentes que protejan dichas inversiones, o bien que invirtió en publicidad y marketing para diferenciar sus productos respecto del de sus competidores, lo cual agregó valor a su producto final. Asimismo, la firma podría sostener argumentaciones basadas en costos hundidos que generan barreras de entrada (competitivas) al mercado, como, por ejemplo, la presencia de inversiones en infraestructura que, al estar hundidas, le generan una ventaja de costos que le permite cobrar precios mayores a su costo marginal. Estos y otros argumentos relacionados con costos ya hundidos son plausibles, en tanto la firma sea capaz de demostrar que están relacionados con el producto o servicio cuyo precio está sometido a análisis.

Una segunda posible justificación, que adelantamos en la nota 72, consiste en argumentar que la firma es una empresa multiproducto, esto es, que comercializa una serie de productos o servicios, de modo que o bien aprovecha economías de ámbito o presenta interdependen- 
cia en las demandas de los bienes que comercializa. Por una parte, desde la perspectiva de las posibles economías de ámbito, se debe analizar cuidadosamente si la firma asigna gran parte de los costos fijos y hundidos al producto cuyo precio se estima excesivo. Lo correcto sería que, dentro del grupo de productos o servicios que comparten costos con aquél cuyo precio ha sido estimado prima facie excesivo, la firma justifique con un criterio económico la asignación de tales costos.

Por otra parte, desde la perspectiva de la interdependencia de las demandas, se debe tener presente que siempre habrá bienes producidos por la firma que serán más necesarios que otros, por lo que la decisión de asignar un precio eficiente debería ser sub-marginalizar aquellos productos que generan más demanda en los otros productos, recuperando de este modo las ganancias con mayores márgenes en esos otros bienes. Tal política comercial puede explicar que un precio real, aun cuando sea mayor al umbral en un caso concreto, pueda no ser considerado excesivo al analizarlo en relación con los demás bienes producidos por la firma. Por esto, en presencia de interdependencia de la demanda, la justificación debiera incluir, por ejemplo, una explicación y cuantificación de las externalidades que se traspasan desde un mercado a otro. ${ }^{81}$

Una situación similar ocurre cuando la empresa multiproducto lanza al mercado un nuevo producto o servicio, pero a pérdida. Lo esperable en este caso es que su estrategia comercial consista en recuperar la pérdida en el futuro cobrando precios por encima de sus costos. Tal situación es plausible y en principio normal en los mercados. En tal caso, la justificación exige que la firma demuestre externalidad de sus demandas en el tiempo. Esta situación se podría dar, por ejemplo, cuando la demanda crece en tanto más consumidores han consumido el producto, o cuando existe un proceso de "aprender haciendo" (learning by doing) en los procesos productivos de la firma, de modo tal que sus costos son más bajos mientras más bienes o servicios haya vendido en el pasado.

Finalmente, una tercera línea de justificaciones, relativamente similar a la anterior, podría estar basada en la externalidad existente entre

${ }^{81}$ Por ejemplo, en lugar de llevar a cabo un análisis producto a producto, el precio promedio para un conjunto de bienes relacionados debería ser comparado con los costos promedio de elaborar ese mismo conjunto de bienes por parte de la empresa superdominante. 
los mercados que la empresa superdominante atiende. En tal caso, la empresa podría ser calificada como una "plataforma" y los mercados, como de "dos-lados" o "multilados". ${ }^{82}$ En tales mercados, la plataforma podría adoptar una política de precios tal que los productos vendidos en un mercado subsidian a los vendidos en otros, por lo que podría ser perfectamente posible observar precios unitarios incluso por encima del umbral en alguno de tales mercados. En esta situación, la plataforma debiera demostrar que sus ingresos promedio para todas sus actividades que presentan la externalidad no exceden el costo promedio en los productos o servicios que ella comercializa. Nótese, sin embargo, que muchas veces las plataformas cobran precios en dos partes; esto es, una tarifa fija más un monto variable por unidad vendida. En tal situación, la firma podría aún demostrar que los retornos promedio resultantes de los precios unitarios que ella cobra no superan sus costos promedio. ${ }^{83}$

\section{CONCLUSIONES}

En este trabajo hemos resaltado la importancia del control de los precios excesivos usando la política de competencia. Ello es especialmente importante en economías altamente desiguales y con ciertas características económicas que hacen difícil la entrada o apertura "natural" de los mercados, como acontece en la mayor parte de los países latinoamericanos, varios de los cuales, lamentablemente, no hacen uso de dicho control. Hemos argumentado, además, que es posible realizarlo de una manera simple y objetiva. Para ello, hemos propuesto un test, o regla en tres etapas, que, en su parte central, combina el análisis de precios y costos de una empresa superdominante, con el fin de establecer un umbral único que permita discernir cuándo un precio debe ser considerado excesivo. Investigaciones futuras pueden explorar en más detalle esta propuesta y su posible aplicación a casos concretos, u otras alternativas que materialicen el control de una manera objetiva.

${ }^{82} \mathrm{La}$ literatura reciente en esta materia es abundante. Por todos, ver Rochet y Tirole (2006) y Evans y Schmalensee (2016).

${ }^{83}$ Una argumentación podría centrarse, por ejemplo, no en los costos, sino en el precio unitario que cobraría una empresa dominante en mercados de dos lados cuya participación fuera del 75 por ciento de mercado. A modo de desafío, dejamos al lector la tarea de encontrar cuál sería el precio de la firma en esta circunstancia. 


\section{REFERENCIAS}

Averitt, Neil W. \& Robert H. Lande. 2007. "Using the 'Consumer Choice' Approach to Antitrust Law". Antitrust Law Journal 74: 175-264.

Baker, Jonathan \& Steven Salop. 2015. "Antitrust, Competition Policy, and Inequality". The Georgetown Law Journal Online 104: 1-28.

Bishop, Simon \& Mike Walker. 2010. The Economics of EC Competition Law: Concepts, Application and Measurement, $3^{\mathrm{a}}$ edición. Londres: Sweet \& Maxwell.

Cahill-O'Callaghan, Rachel. 2013. "The Influence of Personal Values on Legal Judgments". Journal of Law and Society 40 (4): 596-623.

Carter, Michael \& Julian Wright. 2003. “Asymmetric Network Interconnection”. Review of Industrial Organization 22 (1): 27-46.

Coloma, Germán. 2009. "The Argentine Competition Law and its Enforcement". En Competition Law and Policy in Latin America, editado por E. Fox \& D. Sokol, capítulo 5. Oxford: Hart Publishers.

Das Nair, Reena. 2008. "Measuring Excessive Pricing as an Abuse of Dominance - An Assessment of the Criteria Used in the Harmony Gold / Mittal Steel Complaint". South African Journal of Economics Management Sciences 11 (3): 279-291.

Davis, Peter \& Eliana Garcés. 2010. Quantitative Techniques for Competition and Antitrust Analysis. Nueva Jersey: Princeton University Press.

Davis, Peter \& Vivek Mani. 2018. "The Law and Economics of Excessive and Unfair Pricing: A Review and a Proposal". The Antitrust Bulletin 63 (4): 399430 .

De León, Ignacio. 2009. An Institutional Assessment of Antitrust Policy: The Latin American Experience. Países Bajos: Wolters Kluwer.

Deneckere, Raymond J. \& Dan Kovenock. 1992. "Price Leadership". The Review of Economic Studies 59 (1): 143-162.

Elhauge, Einer \& Damien Geradin. 2011. Global Antitrust Law and Economics, $2^{\mathrm{a}}$ edición. Oxford \& Portland, Oregon: Hart.

Evans, David \& Jorge Padilla. 2005. "Excessive Prices: Using Economics to Define Administrable Legal Rules". Journal of Competition Law and Economics 1 (1): 97-122.

Evans, David \& Richard Schmalensee. 2016. Matchmakers. The New Economics of Multisided Platforms. Cambridge: Harvard Business Review Press.

Ezrachi, Ariel \& David Gilo. 2009. “Are Excessive Prices Really Self-Correcting?”. Journal of Competition Law and Economics 5 (2): 249-68.

Fumagalli, Chiara, Massimo Motta \& Claudio Calcagno. 2018 Exclusionary Practices. Nueva York: Cambridge University Press.

Gal, Michal. 2004. "Monopoly Pricing as an Antitrust Offense in the U.S. and the EC: Two Systems of Belief about Monopoly?". Antitrust Bulletin 49: 343-84. 
Hawk, Barry. 2018. “English Competition Law Before 1900”. Antitrust Bulletin 63 (3): 350-374.

Hou, Liyang. 2011. "Excessive Prices within EU Competition Law". European Competition Journal 7 (1): 47-70.

Jenny, Frédéric. 2018. "Abuse of Dominance by Firms Charging Excessive or Unfair Prices: An Assesment". En Excessive Pricing and Competition Law Enforcement, editado por Y. Katsoulacos \& F. Jenny. Berlín, Heidelberg, Dordrecht \& Nueva York: Springer.

Joskow, Paul. 2010. "Regulation of Natural Monopolies". En Handbook of Law and Economics, editado por M. Polinsky \& S. Shavell, capítulo 16. Amsterdam: Elsevier.

Kahai, Simran K., David L. Kaserman \& John W. Mayo. 1996. "Is the 'Dominant Firm' Dominant? An Empirical Analysis of AT\&T's Market Power”. Journal of Law and Economics 39: 499-517.

Kaplow, Louis \& Carl Shapiro. 2010. "Antitrust”. En Handbook of Law and Economics, editado por M. Polinsky y S. Shavell, capítulo 15. Amsterdam: Elsevier.

Katsoulacos, Yannis \& Frédéric Jenny, Eds. 2018. Excessive Pricing and Competition Law Enforcement. Berlín, Heidelberg, Dordrecht \& Nueva York: Springer.

Landes, William \& Richard Posner. 1981. "Market Power in Antitrust Cases". Harvard Law Review 94: 937-996.

Leslie, Will. 2018. "The CJEU's Ruling in AKKA / LAA on Excessive Pricing: One Small Step Forward on a Long Road?". CPI Europe Column, marzo.

Lyons, Bruce. 2007. "The Paradox of the Exclusion of Exploitative Abuse". En The Pros and Cons of High Prices, the Pros and Cons series, Konkurrensverket (Agencia de Competencia de Suecia), capítulo 4.

Mancero, Xavier \& Eduardo Saavedra. 2006. "Un modelo de entrada y competencia en telecomunicaciones". Revista de Análisis Económico 21 (1): 29-57.

Márquez, Pablo. 2012. "Exploitative Excessive Pricing Policy for Emerging Economies: The Case of Latin American Economies". En Temas Atuais de Direito da Concorrencia, editado por A. P. Martínez, capítulo 7. Barcelona: Editora Singular.

Márquez, Pablo \& O. Mutis. 2018. "Precios excesivos en la industria aeroportuaria: la experiencia colombiana". Manuscrito en poder de los autores (en prensa, Universidad del Rosario).

Marshall, Alfred. (1890) 1997. Principles of Economics. Nueva York: Prometheus Books.

Martínez, Ana Paula. 2013. “Abuse of Dominance: The Third Wave of Brazil's Antitrust Enforcement?”. Competition Law International 9 (2): 169-181.

Menchaca, Tomás. 2011. “¿Se debe sancionar la fijación unilateral de precios excesivos?”. En La Libre Competencia en el Chile del Bicentenario. Santiago: Tribunal de Defensa de la Libre Competencia - Thomson Reuters. 
Monsalve, Fabio. 2014. "Scholastic Just Price versus Current Market Price: Is it Merely a Matter of Labelling?". Journal of History Economic Thought 21: 4-20.

Motta, Massimo. 2004. Competition Policy: Theory and Practice. Cambridge: Cambridge University Press.

Motta, Massimo \& Alex de Streel. 2007. "Excessive Pricing in Competition Law: Never Say Never?". En The Pros and Cons of High Prices, the Pros and Cons series, Konkurrensverket (Agencia de Competencia de Suecia), capítulo 2.

O'Donoghue, Robert \& Jorge Padilla. 2013. The Law and Economics of Article 102 TFEU, $2^{\text {a }}$ edición. Oxford: Hart Publishing.

Ono, Yoshiyasu. 1982. "Price Leadership: A Theoretical Analysis". Economica 49 (193): 11-20.

Organización para la Cooperación y el Desarrollo Económicos (OECD). 2011. "Excessive Prices". DAFT/COMP 18.

Posner, Richard. 1976. Antitrust Law: An Economic Perspective. Chicago: University of Chicago Press.

Rassenti, Stephen J. \& Bart J. Wilson. 2004. "How Applicable is the Dominant Firm Model of Price Leadership?". Experimental Economics 7 (3): 271-288.

Roberts, Simon. 2008. "Assessing Excessive Pricing: The Case of Flat Steel in South Africa". Journal of Competition Law and Economics 4 (3): 871-891.

Rochet, Jean-Charles \& Jean Tirole. 2006. "Two-Sided Markets: A Progress Report”. RAND Journal of Economics 37 (3): 645-667.

Saavedra, Eduardo \& Javier Tapia. 2017. "Excessive Pricing: Towards a Workable and Objective Rule". CPI Antitrust Chronicle, August.

Salazar, Andrés. 2016. "La alteración de precios como fraude. Comentarios acerca del origen histórico del artículo 285 del Código Penal chileno y su interpretación". Política Criminal 11 (22): 391-438.

Schumpeter, Joseph. (1911) 1982. The Theory of Economic Development. Londres: Transaction Publishers.

. (1954) 1996. History of Economic Analysis (edición revisada). Oxford: Oxford University Press.

Tapia, Javier. 2014. “'Dime de qué presumes y te diré de qué careces’: E1 tratamiento jurisprudencial de los abusos de dominancia en Chile". Revista de Derecho de la Competencia 10 (10): 47-119.

Tirole, Jean. 1988. The Theory of Industrial Organization. Cambridge, Massachusetts: The MIT Press.

Valdés, Domingo. 1992. La discriminación arbitraria en el derecho económico. Santiago: LexisNexis.

Vásquez, Omar. 2015. "Excessive Pricing: A View from Chile". Working Paper $C C L P(L)$ 41. Center for Competition Law and Policy, Oxford University. EP 